\title{
Goodness-of-fit tests for a heavy tailed distribution
}

\author{
Alex J. Koning* $\quad$ Liang Peng ${ }^{\dagger}$
}

Econometric Institute Report 2005-44

\begin{abstract}
For testing whether a distribution function is heavy tailed, we study the Kolmogorov test, Berk-Jones test, score test and their integrated versions. A comparison is conducted via Bahadur efficiency and simulations.

The score test and the integrated score test show the best performance. Although the Berk-Jones test is more powerful than the Kolmogorov-Smirnov test, this does not hold true for their integrated versions; this differs from results in Einmahl and McKeague (2003), which shows the difference of BerkJones test in testing distributions and tails.
\end{abstract}

Keywords. Bahadur efficiency, heavy tail, tail index.

\section{Contents}

1 Introduction 2

2 Methodologies 4

2.1 The KS, BJ and SC supremum tests . . . . . . . . . . . 4

2.2 Bahadur efficiency . . . . . . . . . . . . . 6

2.3 The KSI, BJI and SCI quadratic tests . . . . . . . . . . 8

3 Simulation study and real applications 9

3.1 Simulation study . . . . . . . . . . . . . . . . 9

3.2 Real applications. . . . . . . . . . . . . . . . 10

4 Proofs

*Econometric Institute, Erasmus University Rotterdam. Email: koning@few.eur.nl

${ }^{\dagger}$ School of Mathematics, Georgia Institute of Technology, Atlanta GA 30332-0160, USA.

Email: peng@math.gatech.edu 


\section{Introduction}

We say that a distribution has a heavy tail with tail index if

$$
\lim _{t \rightarrow \infty} \frac{1-F(t x)}{1-F(t)}=x^{-\alpha} \quad \text { for all } x>0
$$

holds for some $\alpha>0$ (notation: $1-F \in R V_{\alpha}$ ). Heavy tailed distributions have been applied in many different areas such as population size (Zipf (1949)), random graph (Reittu and Norros (2004)), internet traffic (Resnick (1997a)), hydrology (Katz et al. (2002)), finance (Danielsson and de Vries (1997)).

Most of the theoretical work on heavy tailed distributions concentrates on estimating the tail index $\alpha$. One of the best known estimators is the Hill estimator

$$
\hat{\alpha}=\left\{k^{-1} \sum_{j=1}^{k} \log X_{n, n-k+j}-\log X_{n, n-k}\right\}^{-1}
$$

(Hill (1975)), which employs a fraction of upper order statistics $X_{n, n-k}, X_{n, n-k+1}$, $\ldots, X_{n, n}$. Several data-driven methods for choosing the sample fraction $k / n$ are proposed in the literature; see Drees and Kaufmann (1998), Danielsson et al. (2001) and Guillou and Hall (2001).

As far as we know, goodness-of-fit testing has not received as much attention as tail index estimation. An interesting aspect of testing goodness-of-fit of the heavy tail distribution is that the null hypothesis provides a description of the heavy tail distribution which is incomplete in the following two aspects:

(i) The tail index is unknown under the null hypothesis, and hence should be estimated. It is well-known that estimation of unknown parameters has an non-neglible effect on the distribution of the test statistics, see Durbin (1973a,b).

(ii) The tail index only describes the tail behaviour of the heavy tail distribution, and thus only gives a partial description of the underlying distribution.

By fitting a generalized Pareto distribution to exceedances over a high threshold, Davison and Smith (1990) employed Kolmogorov and Anderson-Darling statistics to test the fit, and compared these statistics to 5\% critical values for testing an exponential distribution with unknown mean. However, using these critical values in this context "is suspect since the exponential distribution is only a submodel of the generalized Pareto distribution, so the true critical points are smaller", see Davison and Smith (1990, p. 414). 
In Choulakian and Stephens (2001) critical values for Cramer-von Mises and Anderson-Darling statistics for testing a generalized Pareto distribution with unknown shape parameter are given.

If $X$ has a generalized Pareto distribution with unknown shape parameter, then $\ln X$ has an exponential distribution with unknown mean, and thus tests of exponentiality may be used to test the generalized Pareto distribution, see Marohn (2002).

Recently, Drees et al. (2004) employed the Cramer-von Mises statistic to test the null hypothesis that a distribution is in the domain of attraction with extreme value index larger than $-1 / 2$. This may be thought as a generalization of Choulakian and Stephens (2001), since a deterministic threshold is replaced by a random threshold in Drees et al. (2004)).

As far as the present authors know, no work has been done on comparing the performance of goodness-of-fit tests for heavy tailed distributions under the alternative hypothesis. In this paper we compare three tests, the Kolmogorov test, the Berk-Jones test and the "estimated score" test.

The Kolmogorov test, Kolmogorov (1933); Smirnov (1948); Durbin (1973a), has a long tradition in statistics, and thus is an obvious choice as long as there are no other tests which clearly perform better.

The Berk-Jones test, Berk and Jones $(1978,1979)$, may be viewed as a nonparametric likelihood test, and was derived for the situation where the null hypothesis completely specifies the distribution of the observations. In this particular situation, the Berk-Jones test was shown to be more efficient, in the sense of Bahadur efficiency, than any weighted Kolmogorov test at any alternative. In $\mathrm{Li}$ (2003) it is argued that the Berk-Jones test should also perform better than the Kolmogorov test in situations where the null hypothesis does not completely specify the distribution of the observations.

In contrast to the Berk-Jones test, the "estimated score" test in Hjort and Koning (2002) was specifically proposed for the situation where parameters are unknown.

We organize this paper as follows. In section 2 we present Kolmogorov test, Berk-Jones test, score test and their integrated versions. Large deviations results for tail empirical processes are obtained as a byproduct of studying Bahadur efficiency. A simulation study and real applications are given in section 3. All proofs are put in section 4 . 


\section{Methodologies}

\subsection{The KS, BJ and SC supremum tests}

In order to motivate our methods, we shall first restrict ourselves to the quintessential example of a heavy tailed distribution, the Pareto distribution. The Pareto distribution is defined by

$$
G(x ; \alpha, \beta)=1-(x / \beta)^{-\alpha} \quad \text { for all } x>\beta
$$

where $\alpha>0$ is a shape parameter, and $\beta>0$ is a scale parameter. For convenience, let $G(x ; \alpha)$ denote $G(x ; \alpha, 1)=1-x^{-\alpha}$.

Let $X_{n, 1} \leq X_{n, 2} \leq \cdots \leq X_{n, n}$ denote an ordered sample of size $n$ drawn from the Pareto distribution with unknown shape and scale parameters $\alpha$ and $\beta$. Choose $1 \leq k \leq n$. Let $R_{k, j}$ denote $X_{n, n-k+j} / X_{n, n-k}$ for $j \leq k$, and note that $R_{k, 1} \leq$ $R_{k, 2} \leq \cdots \leq R_{k, k}$. One may show that the random variables $R_{k, 1}, R_{k, 2}, \ldots, R_{k, k}$ are jointly equal in distribution to an ordered sample of size $k$ drawn from the Pareto distribution with shape parameter $\alpha$ and scale parameter 1 .

Let $F_{k}(r)$ denote the empirical distribution function of $R_{k, 1}, R_{k, 2}, \ldots, R_{k, k}$, defined by

$$
F_{k}(r)=k^{-1} \sum_{j=1}^{k} I\left(R_{k, j} \leq r\right) .
$$

Observe that for $r>1$

$$
1-F_{k}(r)=\frac{1}{k} \sum_{i=1}^{n} I\left(X_{i}>r X_{n, n-k}\right) .
$$

The Kolmogorov test is based on the statistic $\sup _{r>1}|\mathrm{KS}(r ; \hat{\alpha})|$, where

$$
\mathrm{KS}(r ; \alpha)=1-F_{k}(r)-\{1-G(r ; \alpha)\}=1-F_{k}(r)-r^{-\alpha},
$$

and $\hat{\alpha}=\left\{k^{-1} \sum_{j=1}^{k} \log R_{k, j}\right\}^{-1}$ is the Hill estimator.

The Berk-Jones test is based on the statistic $\sup _{r>1} k \mathrm{BJ}(r ; \hat{\alpha})$, where

$$
\mathrm{BJ}(r ; \alpha)=2 K\left(F_{k}(r), G(r ; \alpha)\right)=2 K\left(F_{k}(r), 1-r^{-\alpha}\right),
$$

and the function $K$ is defined by

$$
K(\hat{p}, p)=\hat{p} \ln \frac{\hat{p}}{p}+(1-\hat{p}) \ln \frac{1-\hat{p}}{1-p} .
$$


The estimated score test is based on the statistic $\sup _{r>1}|\operatorname{SC}(r ; \hat{\alpha})|$, where

$$
\mathrm{SC}(r ; \alpha)=F_{k}(r)-\int_{1}^{r}\left(1-F_{k}(s)\right) d \Lambda(s ; \alpha)=F_{k}(r)-\alpha \int_{1}^{r} \frac{1-F_{k}(s)}{s} d s,
$$

and $\Lambda(r ; \alpha)=-\ln (1-G(r ; \alpha))=\alpha \ln r$ is the cumulative hazard function belonging to $G(r ; \alpha)$ defined by (2.1).

Suppose that $X_{1}, \cdots, X_{n}$ are i.i.d. observations with distribution function $F$. We intend to test whether $F$ has a heavy tailed distribution, see (1.1). Let $U(x)$ denote the inverse function of $1 /(1-F(x))$. Then (1.1) implies $U \in R V_{1 / \alpha}$. In order to study the limiting behaviors of $\operatorname{KS}(r ; \alpha), \operatorname{BJ}(r ; \alpha)$ and $\operatorname{SC}(r ; \alpha)$, we further assume that there exists a function $A(t) \rightarrow 0$, as $t \rightarrow \infty$, such that

$$
\lim _{t \rightarrow \infty} \frac{U(t x) / U(t)-x^{1 / \alpha}}{A(t)}=x^{1 / \alpha} \frac{x^{\rho}-1}{\rho}
$$

for all $x>0$, where $\rho \leq 0$; see de Haan and Stadtmüller (1996) for details. Our first result is as follows.

Theorem 1. Suppose F satisfies (2.3) and $k=k(n) \rightarrow \infty, k / n \rightarrow 0, \sqrt{k} A(n / k) \rightarrow$ 0 as $n \rightarrow \infty$. Then

$$
\begin{aligned}
& \sup _{x>1}|\sqrt{k} K S(x ; \hat{\alpha})| \\
& \stackrel{d}{\rightarrow} \sup _{0<x<1}\left|W(x)-x W(1)+x \log (x) \int_{0}^{1} \frac{W(s)-s W(1)}{s} d s\right|
\end{aligned}
$$

and

$$
\begin{aligned}
& \sup _{x>1}|\sqrt{k} S C(x ; \hat{\alpha})| \\
& \quad \stackrel{d}{\rightarrow} \sup _{0<x<1}\left|W(x)-x W(1)-\int_{0}^{x} \frac{W(s)-s W(1)}{s} d s+x \int_{0}^{1} \frac{W(s)-s W(1)}{s} d s\right|,
\end{aligned}
$$

where $W(x)$ is a Wiener process.

Remark 1. For any fixed $x^{-1 / \alpha} \in(0,1)$, we can show by Taylor expansions that

$$
B J\left(x^{-1 / \alpha} ; \hat{\alpha}\right) \stackrel{d}{\rightarrow} \frac{\left\{W(x)-x W(1)+x \log (x) \int_{0}^{1} \frac{W(s)-s W(1)}{s} d s\right\}^{2}}{x(1-x)}
$$

and

$$
E \frac{\left\{W(x)-x W(1)+x \log (x) \int_{0}^{1} \frac{W(s)-s W(1)}{s} d s\right\}^{2}}{x(1-x)}=\frac{x(1-x)-x^{2}\{\log (x)\}^{2}}{x(1-x)},
$$


i.e., the expectation of the limiting of $B J(x ; \hat{\alpha})$ depends on location $x$. This is different from Berk \& Jones test for testing distribution functions. But it may still be interesting to find the limiting distribution of $a_{n} \sup _{x>1} B J(x ; \hat{\alpha})-b_{n}$ for some normalizing constants $a_{n}>0$ and $b_{n}$. When $F(x)=\exp \left\{-x^{-\alpha}\right\}$, we have

$$
\begin{aligned}
& \frac{1}{k} \sum_{i=1}^{n} I\left(X_{i}>y^{-1 / \alpha} X_{n, n-k}\right) \\
& \stackrel{d}{=} \frac{1}{k} \sum_{i=1}^{k} I\left(-\alpha \log \frac{U\left(Y_{n, n-i+1}\right)}{U\left(Y_{n, n-k}\right)}<\log y\right) \\
& =\frac{1}{k} \sum_{i=1}^{k} I\left(\log \frac{\log \left(1-Y_{n, n-i+1}^{-1}\right)}{\log \left(1-Y_{n, n-k}^{-1}\right)}<\log y\right),
\end{aligned}
$$

where $Y_{n, i}^{\prime}$ s are defined in the proof of Theorem 1 below. That is, the distributions of

$$
\sup _{x>1}|\sqrt{k} K S(x ; \hat{\alpha})|, \quad \sup _{x>1} B S(x ; \hat{\alpha}) \quad \text { and } \quad \sup _{x>1}|\sqrt{k} S C(x ; \hat{\alpha})|
$$

are independent of $\alpha$ when $F(x)=\exp \left\{-x^{-\alpha}\right\}$. This property is employed to simulate critical values from test statistics themselves; see Section 3 for details.

\subsection{Bahadur efficiency}

It is known that when the null hypothesis is simple, that is, completely specifies the distribution of the observations, the Berk-Jones test is more efficient, in Bahadurs sense, than any weighted KolmogorovSmirnov test at any alternative, see Berk and Jones (1978, 1979).

Extending this result to the situation where the null hypothesis is composite, that is, does not completely specify the distribution of the observations, requires hard work, which is typically avoided. Instead, it is argued that "one may expect similar optimal properties for our test for the composite hypothesis", see for instance Li (2003, p. 178).

In this subsection, we adopt a similar approach. That is, we study the Bahadur efficiency of the "simple null hypothesis tests" based on statistics

$$
\sup _{r>1} k \mathrm{BJ}\left(r ; \alpha_{0}\right) \quad \text { and } \sup _{r>1}\left|\sqrt{k} \mathrm{KS}\left(r ; \alpha_{0}\right)\right|
$$

rather than the Bahadur efficiency of "composite null hypothesis tests" based on the statistics

$$
\sup _{r>1} k \mathrm{BJ}(r ; \hat{\alpha}) \quad \text { and } \quad \sup _{r>1}|\sqrt{k} \mathrm{KS}(r ; \hat{\alpha})| \text {. }
$$


First we state the definition of exact slope of a test statistic. Let $(S, \mathcal{A})$ be the sample space of infinitely many independent and identically distributed observations $s=\left(X_{1}, X_{2}, \cdots\right)$ on an abstract random variable $X$. Let $\left\{P_{\theta}: \theta \in \Omega\right\}$ be a collection of probability distributions of $X$, where $\Omega$ is a parameter space, and let $\Omega_{0}$ be a proper subset of $\Omega$. Consider the hypothesis $H_{0}: \theta \in \Omega_{0}$. Let $\left\{T_{n}\left(X_{1}, \cdots, X_{n}\right)\right\}$ be a sequence of test statistics. Assume that there exists an $F_{n}(t)$ such that

$$
P_{\theta}\left(T_{n} \leq t\right)=F_{n}(t)
$$

for all $\theta \in \Omega_{0}$ and all $t \in R$. Then the level obtained by $T_{n}$ is defined to be

$$
L_{n}\left(X_{1}, \cdots, X_{n}\right)=1-F_{n}\left(T_{n}\left(X_{1}, \cdots, X_{n}\right)\right) .
$$

If

$$
\lim _{n \rightarrow \infty} n^{-1} \log L_{n}\left(X_{1}, \cdots, X_{n}\right)=-\frac{1}{2} c(\theta) \quad \text { a.s. } P_{\theta},
$$

then we say the sequence $\left\{T_{n}\right\}$ has exact slope $c(\theta)$ when $\theta$ obtains.

The following proposition describes a useful method of finding the exact slope of a given sequence $\left\{T_{n}\right\}$ for which (2.4) holds; see Bahadur (1971) for a detailed proof.

Proposition 1. Suppose that

$$
\lim _{n \rightarrow \infty} n^{-1 / 2} T_{n}\left(X_{1}, \cdots, X_{n}\right)=b(\theta) \quad \text { a.s. } P_{\theta}
$$

for each $\theta \in \Omega_{1}=\Omega \backslash \Omega_{0}$, where $-\infty<b(\theta)<\infty$, and that

$$
\lim _{n \rightarrow \infty} n^{-1} \log \left\{1-F_{n}\left(n^{1 / 2} t\right)\right\}=-f(t)
$$

for each $t$ in an open interval $A$, where $f$ is a continuous function on $A$, and $\left\{b(\theta): \theta \in \Omega_{1}\right\} \subset A$. Then (2.5) holds with $c(\theta)=2 f(b(\theta))$ for each $\theta \in \Omega_{1}$.

Now we are ready to give our results on Bahadur efficiency. Let $\Omega_{0}=\{F$ : $\left.1-F \in R V_{\alpha_{0}}\right\}, \Omega_{1}=\left\{F: 1-F \in R V_{\alpha}, \alpha>0, \alpha \neq \alpha_{0}\right\}$ and $\Omega=\Omega_{0} \cup \Omega_{1}$, where $\alpha_{0}>0$ is given. Then we have the following result.

Theorem 2. Suppose $k=k(n) \rightarrow \infty, k / n \rightarrow 0$ and $k / \log \log n \rightarrow \infty$ as $n \rightarrow \infty$. Then

$$
\lim _{n \rightarrow \infty} \sup _{r>1}\left|K S\left(r ; \alpha_{0}\right)\right|=\left|\left(\frac{\alpha_{0}}{\alpha}\right)^{-\frac{\alpha}{\alpha_{0}-\alpha}}-\left(\frac{\alpha_{0}}{\alpha}\right)^{-\frac{\alpha_{0}}{\alpha_{0}-\alpha}}\right| \quad \text { a.s. }
$$

for each $F \in \Omega_{1}$ and that, for $a \in(0,1)$,

$$
\lim _{n \rightarrow \infty} k^{-1} \log P_{\Omega_{0}}\left\{\sup _{r>1}\left|K S\left(r ; \alpha_{0}\right)\right| \geq a\right\}=-f(a),
$$


where $f(a)=\inf \left\{f_{1}(a, t): 0 \leq t \leq 1\right\}$ and

$$
f_{1}(a, t)=\left\{\begin{array}{cl}
(a+t) \log \frac{a+t}{t}+(1-a-t) \log \frac{1-a-t}{1-t} & \text { for } 0 \leq t \leq 1-a, \\
\infty & \text { for } t>1-a .
\end{array}\right.
$$

Remark 2. Using the same arguments as in Berk and Jones (1979), we conclude by Theorem 2 that $\sup _{x>1}\left|k B J\left(r ; \alpha_{0}\right)\right|$ is more Bahadur efficient than $\sup _{r>1}$ $\left|\sqrt{k} K S\left(r ; \alpha_{0}\right)\right|$ for testing $H_{0}: F \in \Omega_{0}$ against $H_{a}: F \in \Omega_{1}$.

Remark 3. It would be interesting to show that (2.6) holds for a larger $\Omega_{1}$. If we replace $\sup _{r>1}\left|\sqrt{k} K S\left(r ; \alpha_{0}\right)\right|$ by $\sup _{r>b}\left|\sqrt{k} K S\left(r ; \alpha_{0}\right)\right|$, where $b>1$ is given, then $\Omega_{1}$ includes all distributions $F$ such that $\lim _{t \rightarrow \infty} \sup _{x>b} \frac{1-F(t x)}{1-F(t)}=0$. In this case, the left hand side of (2.6) becomes $\sup _{x>b} x^{-\alpha_{0}}=b^{-\alpha_{0}}$.

\subsection{The KSI, BJI and SCI quadratic tests}

The KS, BJ and SC tests introduced in paragraph 2.1 are supremum tests, which are based on statistics obtained by taking the supremum over some underlying goodness-of-fit process. Quadratic tests are popular alternatives to supremum tests, and are based on statistics obtained by integrating the squared goodnessof-fit process with respect to some appropriate measure. In this subsection we consider "quadratic" variants of the KS, BJ and SC tests.

The Cramer-von Mises test statistic KSI is defined by

$$
\mathrm{KSI}=\int_{1}^{\infty}\{\sqrt{k} \mathrm{KS}(r ; \hat{\alpha})\}^{2} d G(r ; \hat{\alpha})
$$

As in Einmahl and McKeague (2003), see also Wellner and Koltchinskii (2003), define the integrated BJ test statistic BJI by

$$
\mathrm{BJI}=\int_{1}^{\infty} k \mathrm{BJ}(r ; \hat{\alpha}) d G(r ; \hat{\alpha})
$$

Finally, define the integrated score test SCI by

$$
\mathrm{SCI}=\int_{1}^{\infty}\{\sqrt{k} \mathrm{SC}(r ; \hat{\alpha})\}^{2} d G(r ; \hat{\alpha}) .
$$

The following theorem gives the limiting distributions of these three test statistics.

Theorem 3. Suppose F satisfies (2.3) and $k=k(n) \rightarrow \infty, k / n \rightarrow 0, \sqrt{k} A(n / k) \rightarrow$ 0 as $n \rightarrow \infty$. Then

$$
K S I \stackrel{d}{\rightarrow} \int_{0}^{1}\left\{W(x)-x W(1)+x \log (x) \int_{0}^{1} \frac{W(s)-s W(1)}{s} d s\right\}^{2} d x
$$




$$
B J I \stackrel{d}{\rightarrow} \int_{0}^{1} \frac{\left\{W(x)-x W(1)+x \log (x) \int_{0}^{1} \frac{W(s)-s W(1)}{s} d s\right\}^{2}}{x(1-x)} d x
$$

and

$S C I \stackrel{d}{\rightarrow} \int_{0}^{1}\left\{W(x)-x W(1)-\int_{0}^{x} \frac{W(s)-s W(1)}{s} d s+x \int_{0}^{1} \frac{W(s)-s W(1)}{s} d s\right\}^{2} d x$

where $W(x)$ and $\hat{\alpha}$ are defined in Theorem 1.

\section{Simulation study and real applications}

\subsection{Simulation study}

First we simulate 100,000 random samples from Frechet distribution $F(x)=$ $\exp \left\{-x^{-1}\right\}$ with sample size $n=1000$, and then compute the test statistics KS = $\sup _{r>1}|\sqrt{k} \operatorname{KS}(r ; \hat{\alpha})|, \mathrm{BJ}=\sup _{r>1} k \mathrm{BJ}(r ; \hat{\alpha}), \mathrm{SC}=\sup _{r>1}|\sqrt{k} S C(r ; \hat{\alpha})|, \mathrm{KSI}$, BJI and SCI for $k=20,30, \cdots, 200$. Based on these computed test statistics, we obtain the 0.95 level critical values; see Table 1.

Using Table 1, we compute the powers of these six test statistics by simulating 10,000 random samples from distributions $1-F(x)=\left\{1+\frac{\alpha \log x}{\delta^{-1}}\right\}^{-\delta^{-1}}$, $(x>1)$, with sample size $n=1000, \alpha=1, k=20,30, \cdots, 200$ and $\delta \sqrt{k}=$ $0.1,0.5, \cdots, 6$; see Tables 2-7. Since the limiting distribution of BJ does not exist, we compare the other five tests by simulating critical values from their corresponding limiting distributions. More specifically, we first simulated 100,000 random samples of Wiener Processes on $[0,1]$ with 1000 equally spaced grid points, and then compute the limitings of the test statistics. The critical values with level 0.95 are 1.338, 0.456, 1.076, 0.220 and 1.313 for SC, SCI, KS, KSI and BJI, respectively. Using these numbers, we compute the powers of those five tests as above, which are reported in Tables 8-12.

We summarize our observations from Tables $2-12$ as follows:

1. When the exact critical values are employed, the SCI test is most powerful for most values of $k$ and $\delta$. And the BJ test is more powerful than the KS test, which coincides with the Bahadur efficiency study in section 2.

2. When the asymptotic critical values are employed, the SCI test is still most powerful for most values of $k$ and $\delta$. But the BJI test is less powerful than the KSI test. This seems contradicting Einmahl and McKeague (2003), where an integrated empirical likelihood test is more powerful than the Cramervon Mises test. Note that the Berk-Jones test is an empirical likelihood 
test when testing distributions. Since the limiting of $k \mathrm{BJ}(r ; \hat{\alpha})$ is not a chisquared distribution (see Remark 1), the BJI test is not an exact integrated empirical likelihood test. This is a difference of Berk-Jones test in testing distribution and tails.

3. The tests with asymptotic critical values are comparable to the corresponding tests with exact critical values when $k$ is small, but are more powerful when $k$ is large. This may be because the asymptotic critical values are obtained from the limiting without taking the bias, introduced by a large $k$, into account.

\subsection{Real applications.}

The first data set we analyzed consists of 2156 Danish fire loss over one million Danish krone from the years 1980 to 1990 inclusive (see Figure 1). The loss figure is a total loss for the event concerned and includes damage to buildings, furnishings and personal property as well as loss of profits. This Danish fire loss data set was analyzed by McNeil (1997) and Resnick (1997b), where the right tail index was confirmed to be between 1 and 2. Here we apply the five tests SC, SCI, KS, KSI and BJI to test whether this data set has a heavy tailed distribution. The critical values obtained from the limiting distributions were employed to compute the test statistics for $k=20,21, \cdots, 2000$ by step 1; see Figure 2. The Hill estimators are plotted against $\mathrm{k}$ in Figure 2 as well. For a large range of $k$, all tests do not reject the heavy tailed hypothesis. However, there is a bit strange pattern around $k=150$.

Ideally, when the heavy tailed hypothesis is true, tests should not reject the null hypothesis for small values of $k$, and reject it for large values of $k$ since the critical values are obtained by ignoring the bias. Moreover there should be one region where decision varies due to the fact that the bias and variance are comparable in this range of $k$. We suspect that this strange pattern in Figure 2 may be due to weak dependence inside the danish fire loss data. Next, we use the first 2150 data points and divide into 215 blocks with 10 points in each block. Then we apply those five tests to the maxima in these blocks, see Figure 3. This figure clear shows the ideal pattern. So, it would be very interesting to investigate how the bias, i.e., $\lim \sqrt{k} A(n / k) \neq 0$, affects the tests and to propose tests for heavy tailed time series.

The second data set we analyzed is the internet traffic data. The Ethernet series used here are part of a data set collected at Bellcore in August of 1989. They correspond to one "normal" hour's worth of traffic, collected every 10 milliseconds, thus resulting in a length of 360,000. This data set measures the number of bytes per unit time; see Figure 4. This data set was first analyzed in Leland et al. 
(1994). We apply those five tests to this data set, which clearly reject the heavy tailed hypothesis; see Figure 5. Next we divide this data set into 600 blocks with 600 points in each block, and apply those five tests the maxima in each blocks; see Figure 6. Figure 6 still rejects the heavy tailed hypothesis. This may be due to the long-range dependence of the internet traffic data. How to test heavy tail distributions for long-range dependent data is quite interesting both theoretically and practically since there exist debates on fitting a heavy tailed distribution or log-normal distribution to internet traffic data.

\section{Proofs}

Proof of Theorem 1. Let $Y_{1}, \cdots, Y_{n}$ be i.i.d. random variables with distribution $1-y^{-1},(y>1)$, and $Y_{n, 1} \leq \cdots \leq Y_{n, n}$ denote the order statistics of $Y_{1}, \cdots, Y_{n}$. Then, it follows from de Haan and Resnick (1998) that

$$
\begin{gathered}
\sqrt{k}\left\{\frac{1}{k} \sum_{i=1}^{n} I\left(Y_{i}>\frac{n}{k x}\right)-x\right\} \stackrel{d}{\rightarrow} W(x), \\
\sqrt{k}\left\{\frac{k}{n} Y_{n, n-[k x]}-x^{-1}\right\} \stackrel{d}{\rightarrow} x^{-2} W(x), \\
\sqrt{k}\left\{\frac{1}{k} \sum_{i=1}^{n} I\left(X_{i}>x X_{n, n-k}\right)-x^{-\alpha}\right\} \stackrel{d}{\rightarrow} W\left(x^{-\alpha}\right)-x^{-\alpha} W(1), \\
\sqrt{k}\{\hat{\alpha}-\alpha\} \stackrel{d}{\rightarrow}-\alpha^{2} \int_{1}^{\infty} s^{-1} W\left(s^{-\alpha}\right) d s+\alpha W(1)
\end{gathered}
$$

in $D[1, \infty)$, where $W(x)$ is a Wiener process. So

$$
\begin{aligned}
& \sqrt{k} K S(x ; \hat{\alpha}) \\
& \quad=\sqrt{k}\left\{\frac{1}{k} \sum_{i=1}^{n} I\left(X_{i}>x X_{n, n-k}\right)-x^{-\alpha}\right\} \\
& +\sqrt{k}\left\{x^{-\alpha}-x^{-\hat{\alpha}}\right\} \\
& \stackrel{d}{\rightarrow} W\left(x^{-\alpha}\right)-x^{-\alpha} W(1)-x^{-\alpha} \log x\left\{\alpha^{2} \int_{1}^{\infty} s^{-1} W\left(s^{-\alpha}\right) d s-\alpha W((\mathbb{H}), 2)\right.
\end{aligned}
$$

in $D[1, \infty)$. Hence

$\sup _{x>1}|\sqrt{k} K S(x ; \hat{\alpha})| \stackrel{d}{\rightarrow} \sup _{0<x<1}\left|W(x)-x W(1)+x \log (x) \int_{0}^{1} \frac{W(s)-s W(1)}{s} d s\right|$. 
Similarly, we have

$$
\begin{aligned}
& \sqrt{k} S C(x ; \hat{\alpha}) \\
& =-\sqrt{k}\left\{\frac{1}{k} \sum_{i=1}^{n} I\left(X_{i}>x X_{n, n-k}\right)-x^{-\alpha}\right\} \\
& -\hat{\alpha} \int_{1}^{x} s^{-1} \sqrt{k}\left\{\frac{1}{k} \sum_{i=1}^{n} I\left(X_{i}>x X_{n, n-k}\right)-s^{-\alpha}\right\} d s \\
& -\sqrt{k} \frac{\hat{\alpha}-\alpha}{\alpha}\left\{x^{-\alpha}-1\right\} \\
& \stackrel{d}{\rightarrow}-W\left(x^{-\alpha}\right)+x^{-\alpha} W(1)+\alpha \int_{x}^{\infty} \frac{W\left(s^{-\alpha}\right)-s^{-\alpha} W(1)}{s} d s \\
& \quad-\alpha x^{-\alpha} \int_{1}^{\infty} \frac{W\left(s^{-\alpha}-s^{-\alpha} W(1)\right.}{s} d s
\end{aligned}
$$

in $D[1, \infty)$. Hence

$$
\begin{aligned}
& \sup _{x>1}|\sqrt{k} S C(x ; \hat{\alpha})| \\
& \quad \stackrel{d}{\rightarrow} \sup _{0<x<1}\left|W(x)-x W(1)-\int_{0}^{x} \frac{W(s)-s W(1)}{s} d s+x \int_{0}^{1} \frac{W(s)-s W(1)}{s} d s\right| .
\end{aligned}
$$

Before we prove theorem 2, we give the following Lemma on large deviations for tail processes, which may be of independent interest. Note that Cheng (1992) gave large deviations results for Hill's estimator.

Lemma 1. Under the condition of Theorem 2, we have, for $0<a<1$,

$$
\begin{gathered}
k^{-1} \log P_{\Omega_{0}}\left(\sup _{x>1} K S\left(x ; \alpha_{0}\right) \geq a\right) \rightarrow-f(a), \\
k^{-1} \log P_{\Omega_{0}}\left(\sup _{x>1}\left\{-K S\left(x ; \alpha_{0}\right)\right\} \geq a\right) \rightarrow-f(a)
\end{gathered}
$$

and

$$
k^{-1} \log P_{\Omega_{0}}\left(\sup _{x>1}\left|K S\left(x ; \alpha_{0}\right)\right| \geq a\right) \rightarrow-f(a) .
$$

Proof. Here we only show the first limit. The other two can be shown in a similar way as proving the first limit and Example 5.3 of Bahadur (1971). Let $U_{1}, \cdots, U_{n}$ be i.i.d. random variables with uniform distribution on $[0,1]$ and $U_{n, 1} \leq \cdots \leq$ $U_{n, n}$ denote the order statistics of $U_{1}, \cdots, U_{n}$. Put $G_{n, k+1}(u)=P\left(U_{n, k+1} \leq u\right)$. By Potters' inequality (see Geluk and de Haan (1987)), for any $\epsilon>0$, there exists $u_{0}>0$ such that, for all $0<u \leq u_{0}$ and $0<s \leq 1$,

$$
(1-\epsilon) s^{-1 / \alpha_{0}+\epsilon} \leq(1-F)^{-}(u s) /(1-F)^{-}(u) \leq(1+\epsilon) s^{-1 / \alpha_{0}-\epsilon} .
$$


Write

$$
\begin{aligned}
& P_{\Omega_{0}}\left(\sup _{x>1} K S\left(x ; \alpha_{0}\right) \geq a\right) \\
& =P_{\Omega_{0}}\left(\sup _{x>1}\left\{\frac{1}{k} \sum_{i=1}^{k} I\left(\frac{(1-F)^{-}\left(U_{n, i}\right)}{(1-F)^{-}\left(U_{n, k+1}\right)}>x\right)-x^{-\alpha_{0}}\right\} \geq a\right) \\
& \quad=\int_{0}^{u_{0}} P_{\Omega_{0}}\left(\sup _{x>1}\left\{\frac{1}{k} \sum_{i=1}^{k} I\left(\frac{(1-F)^{-}\left(U_{n, i}\right)}{(1-F)^{-}(u)}>x\right)-x^{-\alpha_{0}}\right\} \geq a \mid U_{n, k+1}=u\right) d G_{n, k+1}(u) \\
& \quad+\int_{u_{0}}^{1} P_{\Omega_{0}}\left(\sup _{x>1}\left\{\frac{1}{k} \sum_{i=1}^{k} I\left(\frac{(1-F)^{-}\left(U_{n, i}\right)}{(1-F)^{-}(u)}>x\right)-x^{-\alpha_{0}}\right\} \geq a \mid U_{n, k+1}=u\right) d G_{n, k+1}(u) \\
& =I_{1}+I_{2} .
\end{aligned}
$$

Let $V_{1}, \cdots, V_{k}$ be i.i.d. random variables with uniform distribution on $[0,1]$. It follows from (4.4) that, for any $x>1$ and $\epsilon \in(0,1)$,

$$
\begin{aligned}
I_{1} & \geq \int_{0}^{u_{0}} P_{\Omega_{0}}\left(\frac{1}{k} \sum_{i=1}^{k} I\left(\frac{(1-F)^{-}\left(U_{n, i}\right)}{(1-F)^{-}(u)}>x\right)-x^{-\alpha_{0}} \geq a \mid U_{n, k+1}=u\right) d G_{n, k+1}(u) \\
& \geq \int_{0}^{u_{0}} P_{\Omega_{0}}\left(\frac{1}{k} \sum_{i=1}^{k} I\left((1-\epsilon)\left(\frac{U_{n, i}}{u}\right)^{-1 / \alpha_{0}+\epsilon}>x\right)-x^{-\alpha_{0}} \geq a \mid U_{n, k+1}=u\right) d G_{n, k+1}(u) \\
& \geq \int_{0}^{u_{0}} P_{\Omega_{0}}\left(\frac{1}{k} \sum_{i=1}^{k} I\left(\frac{U_{n, i}}{u}<\left(\frac{x}{1-\epsilon}\right)^{-\frac{\alpha_{0}}{1-\alpha_{0} \epsilon}}\right)-x^{-\alpha_{0}}-a \geq 0 \mid U_{n, k+1}=u\right) d G_{n, k+1}(u) \\
& =\int_{0}^{u_{0}} P_{\Omega_{0}}\left(\frac{1}{k} \sum_{i=1}^{k} I\left(V_{i}<\left(\frac{x}{1-\epsilon}\right)^{-\frac{\alpha_{0}}{1-\alpha_{0} \epsilon}}\right)-x^{-\alpha_{0}}-a \geq 0\right) d G_{n, k+1}(u) .
\end{aligned}
$$

By Theorem 3.1 of Bahadur (1971),

$$
\begin{aligned}
& \liminf _{n \rightarrow \infty} k^{-1} \log \left(I_{1}\right) \\
& \quad \geq \liminf _{n \rightarrow \infty} k^{-1} \log P_{\Omega_{0}}\left(\frac{1}{k} \sum_{i=1}^{k} I\left(V_{i}<\left(\frac{x}{1-\epsilon}\right)^{-\frac{\alpha_{0}}{1-\alpha_{0} \epsilon}}\right)-x^{-\alpha_{0}}-a \geq 0\right) \\
& \quad+\liminf _{n \rightarrow \infty} k^{-1} \log G_{n, k+1}\left(u_{0}\right) \\
& \geq-f_{1}\left(x^{-\alpha_{0}}-x^{-\frac{\alpha_{0}}{1-\alpha_{0} \epsilon}}+a,\left(\frac{x}{1+\epsilon}\right)^{-\frac{\alpha_{0}}{1-\alpha_{0} \epsilon}}\right)+\liminf _{n \rightarrow \infty} k^{-1} \log G_{n, k+1}\left(u_{0}\right) .
\end{aligned}
$$

Let $\epsilon \rightarrow 0$ and take infinimum over $x>1$, we get

$$
\liminf _{n \rightarrow \infty} k^{-1} \log \left(I_{1}\right) \geq-f(a)+\liminf _{n \rightarrow \infty} k^{-1} \log G_{n, k+1}\left(u_{0}\right) .
$$


Since $U_{n, k+1} \stackrel{p}{\rightarrow}$ 0, i.e., $G_{n, k+1}\left(u_{0}\right) \rightarrow 1$, we have

$\liminf _{n \rightarrow \infty} k^{-1} \log P_{\Omega_{0}}\left(\sup _{x>1} K S\left(x ; \alpha_{0}\right) \geq a\right) \geq \liminf _{n \rightarrow \infty} k^{-1} \log \left(I_{1}\right) \geq-f(a)$.

For any positive integer $m$, define $\Delta(m)=\inf \left\{\frac{(j-1)^{\alpha}{ }^{\alpha}-j^{\alpha_{0}}}{m^{\alpha} 0}: j=1, \cdots, m\right\}$. Then $\Delta(m) \rightarrow 0$ as $m \rightarrow \infty$. Since

$$
\begin{gathered}
\sup _{m / j \leq x \leq m /(j-1)}\left\{\frac{1}{k} \sum_{i=1}^{k} I\left(\frac{(1-F)^{-}\left(U_{n, i}\right)}{(1-F)^{-}(u)}>x\right)-x^{-\alpha_{0}}\right\} \\
\leq \frac{1}{k} \sum_{i=1}^{k} I\left(\frac{(1-F)^{-}\left(U_{n, i}\right)}{(1-F)^{-}(u)}>\frac{m}{j}\right)-\left(\frac{m}{j-1}\right)^{-\alpha_{0}}
\end{gathered}
$$

for $j=1, \cdots, m$, we have, for $0<u \leq u_{0}$

$$
\begin{aligned}
& P\left(\sup _{x>1}\left\{\frac{1}{k} \sum_{i=1}^{k} I\left(\frac{(1-F)^{-}\left(U_{n, i}\right)}{(1-F)^{-}(u)}>x\right)-x^{-\alpha_{0}}\right\} \geq a \mid U_{n, k+1}=u\right) \\
& \quad \leq \sum_{j=1}^{m} P\left(\frac{1}{k} \sum_{i=1}^{k} I\left(\frac{(1-F)^{-}\left(U_{n, i}\right)}{(1-F)^{-}(u)}>\frac{m}{j}\right)-\left(\frac{m}{j-1}\right)^{-\alpha_{0}} \geq a \mid U_{n, k+1}=u\right) \\
& \quad \leq \sum_{j=1}^{m} P\left(\frac{1}{k} \sum_{i=1}^{k} I\left(\frac{U_{n, i}}{u}<\left(\frac{m}{j(1+\epsilon)}\right)^{-\frac{\alpha_{0}}{1+\alpha_{0} \epsilon}}\right)-\left(\frac{m}{j-1}\right)^{-\alpha_{0}}-a \geq 0 \mid U_{n, k+1}=u\right) \\
& \quad=\sum_{j=1}^{m} P\left(\frac{1}{k} \sum_{i=1}^{k} I\left(V_{i}<\left(\frac{m}{j(1+\epsilon)}\right)^{-\frac{\alpha_{0}}{1+\alpha_{0} \epsilon}}\right)-\left(\frac{m}{j-1}\right)^{-\alpha_{0}}-a \geq 0\right) .
\end{aligned}
$$

Choose $m$ large enough such that $0<\left(\frac{m}{j-1}\right)^{-\alpha_{0}}-\left(\frac{m}{j(1+\epsilon)}\right)^{-\frac{\alpha_{0}}{1+\alpha_{0} \epsilon}}<1$ for all $0<\epsilon<1$ and $j=1, \cdots, m$. By Theorem 2.1 of Bahadur (1971), we have

$$
\begin{aligned}
& P\left(\frac{1}{k} \sum_{i=1}^{k} I\left(V_{i}<\left(\frac{m}{j(1+\epsilon)}\right)^{-\frac{\alpha_{0}}{1+\alpha_{0} \epsilon}}\right)-\left(\frac{m}{j-1}\right)^{-\alpha_{0}}-a \geq 0\right) \\
& \quad \leq \exp \left\{-k f_{1}\left(\left(\frac{m}{j-1}\right)^{-\alpha_{0}}-\left(\frac{m}{j(1+\epsilon)}\right)^{-\frac{\alpha_{0}}{1+\alpha_{0} \epsilon}}+a,\left(\frac{m}{j(1+\epsilon)}\right)^{-\frac{\alpha_{0}}{1+\alpha_{0} \epsilon}}\right)\right\}
\end{aligned}
$$

i.e.,

$$
\begin{aligned}
& P\left(\sup _{x>1}\left\{\frac{1}{k} \sum_{i=1}^{k} I\left(\frac{(1-F)^{-}\left(U_{n, i}\right)}{(1-F)^{-}(u)}>x\right)-x^{-\alpha_{0}}\right\} \geq a \mid U_{n, k+1}=u\right) \\
& \leq \sum_{j=1}^{m} \exp \left\{-k f_{1}\left(\left(\frac{m}{j-1}\right)^{-\alpha_{0}}-\left(\frac{m}{j(1+\epsilon)}\right)^{-\frac{\alpha_{0}}{1+\alpha_{0} \epsilon}}+a,\left(\frac{m}{j(1+\epsilon)}\right)^{-\frac{\alpha_{0}}{1+\alpha_{0} \epsilon}}\right)\right\} .
\end{aligned}
$$


Letting $\epsilon \rightarrow 0$, we obtain that

$$
\begin{aligned}
& P\left(\sup _{x>1}\left\{\frac{1}{k} \sum_{i=1}^{k} I\left(\frac{(1-F)^{-}\left(U_{n, i}\right)}{(1-F)^{-}(u)}>x\right)-x^{-\alpha_{0}}\right\} \geq a \mid U_{n, k+1}=u\right) \\
& \quad \leq \sum_{j=1}^{m} \exp \left\{-k f_{1}\left(\left(\frac{m}{j-1}\right)^{-\alpha_{0}}-\left(\frac{m}{j}\right)^{-\alpha_{0}}+a,\left(\frac{m}{j}\right)^{-\alpha_{0}}\right)\right\} \\
& \quad \leq m \exp \left\{-k f_{1}\left(a+\Delta(m),\left(\frac{m}{j}\right)^{-\alpha_{0}}\right)\right\} \\
& \quad \leq m \exp \{-k f(a+\Delta(m))\},
\end{aligned}
$$

i.e.,

$$
I_{1} \leq m \exp \{-k f(a+\Delta(m))\} .
$$

By Hoeffding's inequality (Hoeffding (1963)) we have

$$
\begin{aligned}
& P\left(U_{n, k+1}>u_{0}\right) \\
& \quad=P\left(\sum_{i=1}^{n} I\left(U_{i}>u_{0}\right) \geq n-k-1\right) \\
& \quad \leq \exp \left\{-2 n\left(u_{0}-\frac{k+1}{n}\right)^{2}\right\} .
\end{aligned}
$$

Hence

$$
\limsup _{n \rightarrow \infty} \frac{1}{k} P\left(U_{n, k+1}>u_{0}\right) m^{-1} \exp \{k f(a+\Delta(m))\}=0,
$$

i.e,

$$
\limsup _{n \rightarrow \infty} k^{-1} \log \left(1+I_{2} / I_{1}\right) \leq \limsup _{n \rightarrow \infty} k^{-1} \log \left\{1+\left(1-G_{n, k+1}\left(u_{0}\right)\right) / I_{1}\right\}=0 .
$$

By (4.7) and (4.8),

$$
\limsup _{n \rightarrow \infty} k^{-1} \log P_{\Omega_{0}}\left(\sup _{x>1} K S\left(x ; \alpha_{0}\right) \geq a\right) \leq-f(a) .
$$

Hence, it follows from (4.6) and (4.9) that

$$
\lim _{n \rightarrow \infty} k^{-1} \log P_{\Omega_{0}}\left(\sup _{x>1} K S\left(x ; \alpha_{0}\right) \geq a\right)=-f(a) .
$$

Proof of Theorem 2. (2.6) follows from the fact that

$$
\lim _{n \rightarrow \infty} \sup _{x>1}\left|K S\left(x ; \alpha_{0}\right)\right|=\sup _{x>1}\left|x^{-\alpha}-x^{-\alpha_{0}}\right| \text { a.s. }
$$

for $F \in \Omega_{1}$, and (2.7) follows from Lemma 1. 
Proof of Theorem 3. Obviously, (2.8) and (2.10) follow from (4.2) and (4.3), respectively. To prove (2.9), we follow the lines of Einmahl and McKeague (2003). For $\epsilon \in(0,1)$, write

$$
\begin{aligned}
& -\int_{1}^{\infty} B J(x ; \hat{\alpha}) d x^{-\hat{\alpha}} \\
& \quad=\int_{0}^{\epsilon} B J\left(x^{-1 / \hat{\alpha}} ; \hat{\alpha}\right) d x+\int_{\epsilon}^{1-\epsilon} B J\left(x^{-1 / \hat{\alpha}} ; \hat{\alpha}\right) d x+\int_{1-\epsilon}^{1} B J\left(x^{-1 / \hat{\alpha}} ; \hat{\alpha}\right) d x \\
& =I_{1}+I_{2}+I_{3} .
\end{aligned}
$$

By (4.1), (4.2) and Taylor expansions, we can show that

$$
I_{2} \stackrel{d}{\rightarrow} \int_{\epsilon}^{1-\epsilon} \frac{\left\{W(x)-x W(1)+x \log (x) \int_{0}^{1} \frac{W(s)-s W(1)}{s} d s\right\}^{2}}{x(1-x)} d x
$$

for any $\epsilon \in(0,1)$. Thus, we only need to show that, as $n \rightarrow \infty$,

$$
I_{1}=O_{p}(\sqrt{\epsilon}) \quad \text { and } \quad I_{3}=O_{p}(\sqrt{\epsilon})
$$

uniformly in $\epsilon \in(0,1 / 2)$. Put $\Delta_{n}(x)=\frac{1}{k} \sum_{i=1}^{n} I\left(X_{i}>x X_{n, n-k}\right)$. Write $I_{1}$ as $I I_{1}+\cdots+I I_{7}$, where

$$
\begin{aligned}
& I I_{1}=-\int_{0}^{\epsilon \wedge\left(\frac{X_{n, n}}{X_{n, n-k}}\right)^{-\hat{\alpha}}} k \log (1-x) d x \\
& I I_{2}=-\int_{\epsilon \wedge\left(\frac{X_{n, n}}{X_{n, n-k}}\right)^{-\hat{\alpha}}}^{\epsilon} I\left(x \geq \Delta_{n}\left(x^{-1 / \hat{\alpha}}\right)\right) 2 k\left\{1-\Delta_{n}\left(x^{-1 / \hat{\alpha}}\right)\right\} \\
& \times\left\{\log \left(1+\frac{x-\Delta_{n}\left(x^{-1 / \hat{\alpha}}\right)}{1-x}\right)-\frac{x-\Delta_{n}\left(x^{-1 / \hat{\alpha}}\right)}{1-x}\right\} d x, \\
& I I_{3}=\int_{\epsilon \wedge\left(\frac{X_{n, n}}{X_{n, n-k}}\right)^{-\hat{\alpha}_{n}}}^{\epsilon} I\left(x<\Delta_{n}\left(x^{-1 / \hat{\alpha}}\right)\right) 2 k\left\{1-\Delta_{n}\left(x^{-1 / \hat{\alpha}}\right)\right\} \\
& \times\left\{\log \left(1+\frac{\Delta_{n}\left(x^{-1 / \hat{\alpha}}\right)-x}{1-\Delta_{n}\left(x^{-1 / \hat{\alpha}}\right)}\right)-\frac{\Delta_{n}\left(x^{-1 / \hat{\alpha}_{n}}\right)-x}{1-\Delta_{n}\left(x^{-1 / \hat{\alpha}}\right)}\right\} d x, \\
& I I_{4}=\int_{\epsilon \wedge\left(\frac{X_{n, n}}{X_{n, n-k}}\right)^{-\hat{\alpha}}}^{\epsilon} I\left(x \geq \Delta_{n}\left(x^{-1 / \hat{\alpha}}\right)\right) 2 k \Delta_{n}\left(x^{-1 / \hat{\alpha}}\right) \\
& \times\left\{\log \left(1+\frac{x-\Delta_{n}\left(x^{-1 / \hat{\alpha}}\right)}{\Delta_{n}\left(x^{-1 / \hat{\alpha}}\right)}\right)-\frac{x-\Delta_{n}\left(x^{-1 / \hat{\alpha}}\right)}{\Delta_{n}\left(x^{-1 / \hat{\alpha}}\right)}\right\} d x,
\end{aligned}
$$




$$
\begin{aligned}
& I I_{5}=-\int_{\epsilon \wedge\left(\frac{X_{n, n}}{X_{n, n-k}}\right)^{-\hat{\alpha}}}^{\epsilon} I\left(x<\Delta_{n}\left(x^{-1 / \hat{\alpha}}\right)\right) 2 k \Delta_{n}\left(x^{-1 / \hat{\alpha}}\right) \\
& \times\left\{\log \left(1+\frac{\Delta_{n}\left(x^{-1 / \hat{\alpha}}\right)-x}{x}\right)-\frac{\Delta_{n}\left(x^{-1 / \hat{\alpha}}\right)-x}{x}\right\} d x, \\
& I I_{6}=-\int_{\epsilon \wedge\left(\frac{X_{n, n}}{X_{n, n-k}}\right)^{-\hat{\alpha}}}^{\epsilon} I\left(x \geq \Delta_{n}\left(x^{-1 / \hat{\alpha}}\right) 2 k \frac{\left(x-\Delta_{n}\left(x^{-1 / \hat{\alpha}}\right)\right)^{2}}{1-x} d x,\right. \\
& I I_{7}=-\int_{\epsilon \wedge\left(\frac{X_{n, n}}{X_{n, n-k}}\right)^{-\hat{\alpha}}}^{\epsilon} I\left(x<\Delta_{n}\left(x^{-1 / \hat{\alpha}}\right)\right) 2 k \frac{\left(\Delta_{n}\left(x^{-1 / \hat{\alpha}}\right)-x\right)^{2}}{x} d x .
\end{aligned}
$$

Let $U_{1}, \cdots, U_{n}$ be i.i.d. random variables with uniform distribution on $[0,1]$, and $U_{n, 1} \leq \cdots \leq U_{n, n}$ denote the order statistics. It follows from Einmahl (1997) that there exists a sequence of Wiener processes $W_{n}$ such that for any $\delta>0$

$$
\sup _{t>0} t^{-1 / 2} e^{-\delta|\log t|}\left|\sqrt{k}\left\{\frac{1}{k} \sum_{i=1}^{n} I\left(U_{i} \leq \frac{k}{n} t\right)-t\right\}-W_{n}(t)\right| \stackrel{p}{\rightarrow} 0
$$

as $n \rightarrow \infty$, where $k=k(n) \rightarrow \infty$ and $k / n \rightarrow 0$ as $n \rightarrow \infty$. Using (4.12) and similar arguments in Drees et al. (2004), we can show that, for any $\delta>0$,

$$
\begin{aligned}
\sup _{0<x \leq \frac{1}{2}} & x^{-1 / 2+\delta} \mid \sqrt{k}\left\{\Delta_{n}\left(x^{-1 / \hat{\alpha}}\right)-x\right\}-W_{n}(x)+x W_{n}(1) \\
& +x \log (x) \int_{0}^{1} \frac{W_{n}(s)-s W_{n}(1)}{s} d s-\sqrt{k} A(n / k) \alpha x \frac{x^{-\rho}-1}{\rho} \mid \stackrel{p}{(403)}
\end{aligned}
$$

as $n \rightarrow \infty$. Note that

$$
|\log (1+y)-y| \leq 2 y^{2} \text { for } y \geq 0
$$

It is easy to check that for any $\delta_{1}>0$ and $\delta_{2}>0$

$$
P\left(k^{-1-\delta_{1}} \leq\left(\frac{X_{n, n}}{X_{n, n-k}}\right)^{-\hat{\alpha}} \leq k^{-1+\delta_{2}}\right) \rightarrow 1
$$

and

$$
P\left(k^{-1-\delta_{1}} \leq 1-\left(\frac{X_{n, n-k+1}}{X_{n, n-k}}\right)^{-\hat{\alpha}} \leq k^{-1+\delta_{2}}\right) \rightarrow 1
$$

as $n \rightarrow \infty$. By (4.15), we have

$$
I I_{1}=O_{p}(\sqrt{\epsilon}) \text { uniformly in } \epsilon \in(0,1 / 2) .
$$


Using (4.13), (4.14) and (4.15), we have

$$
\begin{aligned}
\left|I I_{5}\right| \leq & \int_{\epsilon \wedge\left(\frac{X_{n, n}}{X_{n, n-k}}\right)^{-\hat{\alpha}}}^{\epsilon} 4 k\left\{1-\Delta_{n}\left(x^{-1 / \hat{\alpha}}\right)\right\}\left\{\frac{x-\Delta_{n}\left(x^{-1 / \hat{\alpha}}\right)}{x}\right\}^{2} d x \\
= & \int_{\epsilon \wedge\left(\frac{X_{n, n}}{X_{n, n-k}}\right)^{-\hat{\alpha}}}^{\epsilon} 4 k \frac{\left(\Delta_{n}\left(x^{-1 / \hat{\alpha}}\right)-x\right)^{3}}{x^{2}} d x \\
& +\int_{\epsilon \wedge\left(\frac{x_{n, n}}{X_{n, n-k}}\right)^{-\hat{\alpha}}}^{\epsilon} \frac{\left(\Delta_{n}\left(x^{-1 / \hat{\alpha}}\right)-x\right)^{2}}{x} d x \\
= & O_{p}\left(I\left(\epsilon>k^{-1-\delta_{1}}\right) \int_{0}^{\epsilon} \frac{1}{\sqrt{k}} \frac{\left(x^{1 / 2-\delta}\right)^{3}}{x^{2}} d x\right) \\
& +O_{p}\left(\int_{0}^{\epsilon} \frac{\left(x^{1 / 2-\delta}\right)^{2}}{x} d x\right) \text { uniformly in } \epsilon \in(0,1 / 2) \\
= & O_{p}(\sqrt{\epsilon}) \text { uniformly in } \epsilon \in(0,1 / 2) .
\end{aligned}
$$

Similarly, we can show that

$$
I I_{i}=O_{p}(\sqrt{\epsilon}) \text { uniformly in } \epsilon \in(0,1 / 2)
$$

for $i=2,3,4,6,7$. Hence

$$
I_{1}=O_{p}(\sqrt{\epsilon}) \text { uniformly in } \epsilon \in(0,1 / 2) .
$$

Similarly, we can show that

$$
I_{3}=O_{p}(\sqrt{\epsilon}) \text { uniformly in } \epsilon \in(0,1 / 2) .
$$

Thus, the theorem follows.

Acknowledgment. Peng's research was partly supported by NSF grant DMS0403443. Alex J. Koning is grateful to the School of Mathematics at the Georgia Institute of Technology for hospitality and financial support.

\section{References}

R. R. Bahadur. Some limit theorems in statistics. Society for Industrial and Applied Mathematics, Philadelphia, Pa., 1971.

Robert H. Berk and Douglas H. Jones. Relatively optimal combinations of test statistics. Scandinavian Journal of Statistics, 5(3):158-162, 1978. 
Robert H. Berk and Douglas H. Jones. Goodness-of-fit test statistics that dominate the Kolmogorov statistics. Zeitschrift für Wahrscheinlichkeitstheorie und Verwandte Gebiete, 47(1):47-59, 1979.

Shi Hong Cheng. Large deviation theorem for Hill's estimator. Acta Mathematica Sinica. New Series, 8(3):243-254, 1992.

V. Choulakian and M. A. Stephens. Goodness-of-fit tests for the generalized Pareto distribution. Technometrics, 43(4):478-484, 2001.

J. Danielsson and C. G. de Vries. Tail index and quantile estimation with very high frequency data. Journal of Empirical Finance, 4(2-3):241-257, June 1997.

J. Danielsson, L. de Haan, L. Peng, and C. G. de Vries. Using a bootstrap method to choose the sample fraction in tail index estimation. Journal of Multivariate Analysis, 76(2):226-248, 2001. doi: 10.1006/jmva.2000.1903.

A. C. Davison and R. L. Smith. Models for exceedances over high thresholds. Journal of the Royal Statistical Society. Series B. Methodological, 52(3):393442, 1990.

Laurens de Haan and Sidney Resnick. On asymptotic normality of the Hill estimator. Communications in Statistics. Stochastic Models, 14(4):849-866, 1998.

Laurens de Haan and Ulrich Stadtmüller. Generalized regular variation of second order. Australian Mathematical Society. Journal. Series A. Pure Mathematics and Statistics, 61(3):381-395, 1996.

Holger Drees and Edgar Kaufmann. Selecting the optimal sample fraction in univariate extreme value estimation. Stochastic Processes and their Applications, 75(2):149-172, 1998.

Holger Drees, Laurens de Haan, and Deyuan Li. Approximations to the tail empirical distribution function with application to testing extreme value conditions. Technical Report 200401, Fachbereich Mathematik, Universität Hamburg, 2004. URL http://www.math.uni-hamburg.de/meta/preprints/ims/prst2004-01.html.

J. Durbin. Distribution theory for tests based on the sample distribution function. Society for Industrial and Applied Mathematics, pages vi+64, Philadelphia, Pa. 1973a.

James Durbin. Weak convergence of the sample distribution function when parameters are estimated. The Annals of Statistics, 1:279-290, 1973b. 
John H. J. Einmahl. Poisson and Gaussian approximation of weighted local empirical processes. Stochastic Processes and their Applications, 70(1):31-58, 1997. doi: 10.1016/S0304-4149(97)00055-0.

John H. J. Einmahl and Ian W. McKeague. Empirical likelihood based hypothesis testing. Bernoulli, 9(2):267-290, 2003.

J. L. Geluk and L. de Haan. Regular variation, extensions and Tauberian theorems, volume 40 of CWI Tract. Stichting Mathematisch Centrum - Centrum voor Wiskunde en Informatica, Amsterdam, 1987. ISBN 90-6196-324-9.

Armelle Guillou and Peter Hall. A diagnostic for selecting the threshold in extreme value analysis. Journal of the Royal Statistical Society. Series B. Statistical Methodology, 63(2):293-305, 2001. doi: 10.1111/1467-9868.00286.

Bruce M. Hill. A simple general approach to inference about the tail of a distribution. The Annals of Statistics, 3(5):1163-1174, 1975.

Nils Lid Hjort and Alexander Koning. Tests for constancy of model parameters over time. Journal of Nonparametric Statistics, 14(1-2):113-132, 2002. doi: 10.1080/10485250211394. Statistical models and methods for discontinuous phenomena (Oslo, 1998).

Wassily Hoeffding. Probability inequalities for sums of bounded random variables. J. Amer. Statist. Assoc., 58:13-30, 1963.

Richard W. Katz, Marc B. Parlange, and Phillipe Naveau. Statistics of extremes in hydrology. Advances in Water Resources, 25:12871304, 2002. doi: 10.1016/ S0309-1708(02)00056-8.

A.N. Kolmogorov. Sulla determinazione empirica di una legge di distribuzione. Giorn. Ist. Ital. Attuari., 4:83-91, 1933.

W. E. Leland, M. S. Taqqu, W. Willinger, and D. V. Wilson. On the self-similar nature of ethernet traffic (extended version). IEEE/ACM Transactions on Networking, 2:1-15, Feb 1994.

Gang Li. Nonparametric likelihood ratio goodness-of-fit tests for survival data. Journal of Multivariate Analysis, 86(1):166-182, 2003. doi: 10.1016/ S0047-259X(02)00058-1.

Frank Marohn. A characterization of generalized Pareto distributions by progressive censoring schemes and goodness-of-fit tests. Communications in Statistics. Theory and Methods, 31(7):1055-1065, 2002. doi: 10.1081/STA-120004902. 
Alexander J. McNeil. Estimating the tails of loss severity distributions using extreme value theory. Astin Bulletin, 27(1):117-137, 1997.

Hannu Reittu and Ilkka Norros. On the power-law random graph model of massive data networks. Performance Evaluation, 55(1-2):3-23, Jan 2004. doi: 10.1016/ S0166-5316(03)00097-X.

Sidney I. Resnick. Heavy tail modeling and teletraffic data. The Annals of Statistics, 25(5):1805-1869, 1997a. doi: 10.1214/aos/1069362376.

Sidney I. Resnick. Discussion of the danish data on large fire insurance losses. Astin Bulletin, 27(1):139-151, 1997b.

N. V. Smirnov. Table for estimating the goodness of fit of empirical distributions. The Annals of Mathematical Statistics, 19(2):279-281, Jun. 1948.

Jon A. Wellner and Vladimir Koltchinskii. A note on the asymptotic distribution of Berk-Jones type statistics under the null hypothesis. In High dimensional probability, III (Sandjberg, 2002), volume 55 of Progr. Probab., pages 321-332. Birkhäuser, Basel, 2003. URL http://www.stat.washington.edu/jaw/RESEARCH/PAPERS/HDP2002-rev.pdf.

G.K. Zipf. Human Behavior and Principle of Least Effort: an Introduction to Human Ecology. Addison Wesley, Cambridge, MA, 1949. 
Table 1: 0.95 -level critical values based on test statistics

\begin{tabular}{|r|r|r|r|r|r|r||}
\hline$k$ & SC & SCI & KS & KSI & BJ & BJI \\
\hline 20 & 1.335 & 0.436 & 1.047 & 0.210 & 6.865 & 1.133 \\
30 & 1.340 & 0.448 & 1.057 & 0.215 & 7.307 & 1.198 \\
40 & 1.339 & 0.448 & 1.062 & 0.217 & 7.575 & 1.230 \\
50 & 1.343 & 0.453 & 1.066 & 0.218 & 7.734 & 1.257 \\
60 & 1.344 & 0.453 & 1.070 & 0.219 & 7.879 & 1.267 \\
70 & 1.346 & 0.454 & 1.071 & 0.220 & 8.042 & 1.278 \\
80 & 1.353 & 0.458 & 1.077 & 0.222 & 8.166 & 1.297 \\
90 & 1.357 & 0.460 & 1.079 & 0.224 & 8.249 & 1.306 \\
100 & 1.358 & 0.464 & 1.079 & 0.223 & 8.348 & 1.310 \\
110 & 1.361 & 0.466 & 1.082 & 0.223 & 8.425 & 1.320 \\
120 & 1.369 & 0.470 & 1.086 & 0.226 & 8.499 & 1.338 \\
130 & 1.375 & 0.473 & 1.090 & 0.228 & 8.586 & 1.347 \\
140 & 1.378 & 0.478 & 1.094 & 0.229 & 8.607 & 1.366 \\
150 & 1.386 & 0.483 & 1.097 & 0.232 & 8.644 & 1.383 \\
160 & 1.392 & 0.489 & 1.100 & 0.234 & 8.746 & 1.394 \\
170 & 1.340 & 0.496 & 1.106 & 0.237 & 8.776 & 1.416 \\
180 & 1.409 & 0.509 & 1.114 & 0.241 & 8.891 & 1.441 \\
190 & 1.421 & 0.516 & 1.119 & 0.245 & 8.943 & 1.459 \\
200 & 1.423 & 0.528 & 1.125 & 0.248 & 9.019 & 1.484 \\
\hline
\end{tabular}


Table 2: Powers for SC with level 0.95 and exact critical values given in Table 1.

\begin{tabular}{|r|r|r|r|r|r|r|r|r|r|r||}
\hline & \multicolumn{10}{|c|}{$\delta \sqrt{k}$} \\
$k$ & 0.1 & 0.5 & 1 & 1.5 & 2 & 2.5 & 3 & 4 & 5 & 6 \\
\hline 20 & 0.0498 & 0.0621 & 0.1119 & 0.2009 & 0.3057 & 0.4179 & 0.5219 & 0.6992 & 0.8179 & 0.8912 \\
30 & 0.0478 & 0.0587 & 0.1166 & 0.2138 & 0.3338 & 0.4535 & 0.5693 & 0.7523 & 0.8663 & 0.9298 \\
40 & 0.0496 & 0.0645 & 0.1183 & 0.2229 & 0.3481 & 0.4789 & 0.5955 & 0.7829 & 0.8945 & 0.9552 \\
50 & 0.0458 & 0.0617 & 0.1196 & 0.2258 & 0.3525 & 0.4882 & 0.6096 & 0.7991 & 0.9098 & 0.9635 \\
60 & 0.0499 & 0.0638 & 0.1205 & 0.2215 & 0.3582 & 0.4997 & 0.6220 & 0.8144 & 0.9218 & 0.9707 \\
70 & 0.0460 & 0.0608 & 0.1207 & 0.2198 & 0.3563 & 0.5006 & 0.6298 & 0.8210 & 0.9273 & 0.9760 \\
80 & 0.0448 & 0.0604 & 0.1196 & 0.2141 & 0.3524 & 0.5002 & 0.6319 & 0.8287 & 0.9344 & 0.9792 \\
90 & 0.0438 & 0.0615 & 0.1180 & 0.2172 & 0.3555 & 0.5044 & 0.6389 & 0.8350 & 0.9391 & 0.9803 \\
100 & 0.0460 & 0.0631 & 0.1195 & 0.2178 & 0.3520 & 0.5033 & 0.6421 & 0.8401 & 0.9425 & 0.9805 \\
110 & 0.0442 & 0.0652 & 0.1169 & 0.2164 & 0.3531 & 0.5028 & 0.6392 & 0.8414 & 0.9462 & 0.9826 \\
120 & 0.0431 & 0.0598 & 0.1132 & 0.2148 & 0.3466 & 0.4953 & 0.6361 & 0.8417 & 0.9472 & 0.9833 \\
130 & 0.0429 & 0.0588 & 0.1121 & 0.2054 & 0.3404 & 0.4945 & 0.6343 & 0.8423 & 0.9497 & 0.9845 \\
140 & 0.0398 & 0.0553 & 0.1092 & 0.2058 & 0.3402 & 0.4907 & 0.6354 & 0.8449 & 0.9511 & 0.9861 \\
150 & 0.0396 & 0.0529 & 0.1030 & 0.2043 & 0.3369 & 0.4818 & 0.6338 & 0.8434 & 0.9503 & 0.9866 \\
160 & 0.0374 & 0.0533 & 0.1053 & 0.1996 & 0.3288 & 0.4785 & 0.6324 & 0.8441 & 0.9490 & 0.9878 \\
170 & 0.0373 & 0.0528 & 0.1019 & 0.1931 & 0.3252 & 0.4741 & 0.6223 & 0.8443 & 0.9482 & 0.9879 \\
180 & 0.0362 & 0.0502 & 0.0982 & 0.1883 & 0.3151 & 0.4643 & 0.6168 & 0.8421 & 0.9496 & 0.9877 \\
190 & 0.0349 & 0.0468 & 0.0918 & 0.1801 & 0.3087 & 0.4576 & 0.6104 & 0.8391 & 0.9469 & 0.9870 \\
200 & 0.0336 & 0.0446 & 0.0904 & 0.1777 & 0.3033 & 0.4471 & 0.6041 & 0.8366 & 0.9473 & 0.9873 \\
\hline
\end{tabular}


Table 3: Powers for SCI with level 0.95 and exact critical values given in Table 1.

\begin{tabular}{|r|r|r|r|r|r|r|r|r|r|r||}
\hline & \multicolumn{10}{|c|}{$\delta \sqrt{k}$} \\
$k$ & 0.1 & 0.5 & 1 & 1.5 & 2 & 2.5 & 3 & 4 & 5 & 6 \\
\hline 20 & 0.0517 & 0.0898 & 0.1762 & 0.2864 & 0.4077 & 0.5258 & 0.6299 & 0.7840 & 0.8757 & 0.9297 \\
30 & 0.0512 & 0.0854 & 0.1718 & 0.2924 & 0.4270 & 0.5524 & 0.6657 & 0.8214 & 0.9123 & 0.9576 \\
40 & 0.0552 & 0.0862 & 0.1717 & 0.2984 & 0.4406 & 0.5725 & 0.6809 & 0.8454 & 0.9330 & 0.9739 \\
50 & 0.0526 & 0.0844 & 0.1704 & 0.3021 & 0.4433 & 0.5792 & 0.6911 & 0.8597 & 0.9430 & 0.9793 \\
60 & 0.0537 & 0.0824 & 0.1674 & 0.2973 & 0.4475 & 0.5885 & 0.7065 & 0.8700 & 0.9519 & 0.9825 \\
70 & 0.0491 & 0.0784 & 0.1648 & 0.2891 & 0.4468 & 0.5891 & 0.7074 & 0.8774 & 0.9583 & 0.9876 \\
80 & 0.0520 & 0.0784 & 0.1605 & 0.2860 & 0.4425 & 0.5909 & 0.7168 & 0.8844 & 0.9599 & 0.9882 \\
90 & 0.0542 & 0.0791 & 0.1631 & 0.2886 & 0.4423 & 0.5907 & 0.7172 & 0.8901 & 0.9631 & 0.9888 \\
100 & 0.0552 & 0.0825 & 0.1577 & 0.2814 & 0.4409 & 0.5895 & 0.7194 & 0.8909 & 0.9640 & 0.9892 \\
110 & 0.0515 & 0.0785 & 0.1568 & 0.2809 & 0.4348 & 0.5877 & 0.7221 & 0.8920 & 0.9685 & 0.9912 \\
120 & 0.0518 & 0.0782 & 0.1553 & 0.2784 & 0.4297 & 0.5862 & 0.7194 & 0.8949 & 0.9689 & 0.9917 \\
130 & 0.0514 & 0.0775 & 0.1494 & 0.2743 & 0.4289 & 0.5819 & 0.7195 & 0.8980 & 0.9702 & 0.9922 \\
140 & 0.0466 & 0.0713 & 0.1482 & 0.2723 & 0.4274 & 0.5825 & 0.7187 & 0.8990 & 0.9720 & 0.9928 \\
150 & 0.0470 & 0.0699 & 0.1436 & 0.2650 & 0.4205 & 0.5793 & 0.7164 & 0.9001 & 0.9715 & 0.9935 \\
160 & 0.0463 & 0.0719 & 0.1463 & 0.2633 & 0.4128 & 0.5755 & 0.7159 & 0.8962 & 0.9738 & 0.9940 \\
170 & 0.0432 & 0.0690 & 0.1393 & 0.2586 & 0.4065 & 0.5674 & 0.7136 & 0.8983 & 0.9723 & 0.9942 \\
180 & 0.0416 & 0.0636 & 0.1319 & 0.2451 & 0.3997 & 0.5593 & 0.7063 & 0.8941 & 0.9716 & 0.9942 \\
190 & 0.0405 & 0.0610 & 0.1270 & 0.2413 & 0.3922 & 0.5518 & 0.7066 & 0.8948 & 0.9720 & 0.9939 \\
200 & 0.0370 & 0.0578 & 0.1229 & 0.2393 & 0.3849 & 0.5453 & 0.7034 & 0.8917 & 0.9707 & 0.9949 \\
\hline
\end{tabular}


Table 4: Powers for KS with level 0.95 and exact critical values given in Table 1.

\begin{tabular}{||r|r|r|r|r|r|r|r|r|r|r||}
\hline & \multicolumn{10}{|c|}{$\delta \sqrt{k}$} \\
$k$ & 0.1 & 0.5 & 1 & 1.5 & 2 & 2.5 & 3 & 4 & 5 & 6 \\
\hline 20 & 0.0521 & 0.0692 & 0.1290 & 0.2180 & 0.3257 & 0.4307 & 0.5434 & 0.7132 & 0.8318 & 0.9364 \\
30 & 0.0510 & 0.0662 & 0.1206 & 0.2125 & 0.3258 & 0.4443 & 0.5639 & 0.7468 & 0.8627 & 0.9299 \\
40 & 0.0519 & 0.0699 & 0.1195 & 0.2097 & 0.3267 & 0.4570 & 0.5747 & 0.7636 & 0.8832 & 0.9469 \\
50 & 0.0490 & 0.0689 & 0.1220 & 0.2136 & 0.3293 & 0.4558 & 0.5774 & 0.7756 & 0.8944 & 0.9562 \\
60 & 0.0537 & 0.0692 & 0.1213 & 0.2105 & 0.3240 & 0.4568 & 0.5890 & 0.7842 & 0.9008 & 0.9649 \\
70 & 0.0508 & 0.0664 & 0.1148 & 0.2063 & 0.3188 & 0.4516 & 0.5871 & 0.7904 & 0.9100 & 0.9682 \\
80 & 0.0493 & 0.0658 & 0.1126 & 0.1957 & 0.3140 & 0.4487 & 0.5842 & 0.7957 & 0.9156 & 0.9698 \\
90 & 0.0480 & 0.0657 & 0.1080 & 0.1972 & 0.3146 & 0.4496 & 0.5827 & 0.7979 & 0.9199 & 0.9716 \\
100 & 0.0459 & 0.0626 & 0.1119 & 0.1929 & 0.3154 & 0.4483 & 0.5821 & 0.8000 & 0.9239 & 0.9717 \\
110 & 0.0462 & 0.0614 & 0.1120 & 0.1933 & 0.3088 & 0.4431 & 0.5833 & 0.7974 & 0.9214 & 0.9746 \\
120 & 0.0480 & 0.0632 & 0.1071 & 0.1879 & 0.3035 & 0.4375 & 0.5768 & 0.7930 & 0.9233 & 0.9763 \\
130 & 0.0454 & 0.0605 & 0.1061 & 0.1814 & 0.2967 & 0.4319 & 0.5705 & 0.7965 & 0.9244 & 0.9767 \\
140 & 0.0428 & 0.0557 & 0.0993 & 0.1770 & 0.2903 & 0.4238 & 0.5638 & 0.7917 & 0.9241 & 0.9789 \\
150 & 0.0416 & 0.0536 & 0.0990 & 0.1771 & 0.2893 & 0.4224 & 0.5610 & 0.7923 & 0.9237 & 0.9771 \\
160 & 0.0406 & 0.0535 & 0.0964 & 0.1762 & 0.2844 & 0.4202 & 0.5563 & 0.7941 & 0.9247 & 0.9781 \\
170 & 0.0378 & 0.0526 & 0.0939 & 0.1694 & 0.2802 & 0.4072 & 0.5488 & 0.7911 & 0.9245 & 0.9772 \\
180 & 0.0380 & 0.0510 & 0.0896 & 0.1618 & 0.2676 & 0.4032 & 0.5439 & 0.7861 & 0.9216 & 0.9764 \\
190 & 0.0378 & 0.0494 & 0.0864 & 0.1570 & 0.2666 & 0.3937 & 0.5337 & 0.7835 & 0.9190 & 0.9771 \\
200 & 0.0378 & 0.0496 & 0.0852 & 0.1511 & 0.2616 & 0.3925 & 0.5296 & 0.7780 & 0.9189 & 0.9763 \\
\hline
\end{tabular}


Table 5: Powers for KSI with level 0.95 and exact critical values given in Table 1.

\begin{tabular}{||r|r|r|r|r|r|r|r|r|r|r||}
\hline & \multicolumn{10}{|c|}{$\delta \sqrt{k}$} \\
$k$ & 0.1 & 0.5 & 1 & 1.5 & 2 & 2.5 & 3 & 4 & 5 & 6 \\
\hline 20 & 0.0512 & 0.0798 & 0.1529 & 0.2527 & 0.3720 & 0.4893 & 0.5940 & 0.7608 & 0.8649 & 0.9222 \\
30 & 0.0520 & 0.0778 & 0.1463 & 0.2519 & 0.3815 & 0.5056 & 0.6254 & 0.7920 & 0.8960 & 0.9493 \\
40 & 0.0553 & 0.0777 & 0.1398 & 0.2522 & 0.3832 & 0.5200 & 0.6350 & 0.8132 & 0.9127 & 0.9652 \\
50 & 0.0497 & 0.0747 & 0.1397 & 0.2545 & 0.3867 & 0.5204 & 0.6447 & 0.8226 & 0.9257 & 0.9734 \\
60 & 0.0535 & 0.0728 & 0.1351 & 0.2486 & 0.3817 & 0.5267 & 0.6521 & 0.8360 & 0.9339 & 0.9766 \\
70 & 0.0463 & 0.0696 & 0.1325 & 0.2373 & 0.3782 & 0.5213 & 0.6508 & 0.8428 & 0.9422 & 0.9809 \\
80 & 0.0498 & 0.0683 & 0.1291 & 0.2330 & 0.3709 & 0.5196 & 0.6536 & 0.8494 & 0.9439 & 0.9830 \\
90 & 0.0504 & 0.0670 & 0.1280 & 0.2341 & 0.3664 & 0.5191 & 0.6524 & 0.8495 & 0.9475 & 0.9830 \\
100 & 0.0496 & 0.0710 & 0.1280 & 0.2317 & 0.3611 & 0.5191 & 0.6552 & 0.8557 & 0.9494 & 0.9837 \\
110 & 0.0522 & 0.0713 & 0.1299 & 0.2265 & 0.3629 & 0.5140 & 0.6574 & 0.8533 & 0.9531 & 0.9854 \\
120 & 0.0497 & 0.0672 & 0.1224 & 0.2239 & 0.3581 & 0.5102 & 0.6538 & 0.8546 & 0.9537 & 0.9873 \\
130 & 0.0480 & 0.0648 & 0.1187 & 0.2156 & 0.3519 & 0.5055 & 0.6469 & 0.8528 & 0.9541 & 0.9878 \\
140 & 0.0428 & 0.0600 & 0.1164 & 0.2156 & 0.3526 & 0.5002 & 0.6438 & 0.8571 & 0.9551 & 0.9888 \\
150 & 0.0428 & 0.0566 & 0.1129 & 0.2106 & 0.3445 & 0.4958 & 0.6456 & 0.8577 & 0.9552 & 0.9889 \\
160 & 0.0425 & 0.0599 & 0.1139 & 0.2059 & 0.3388 & 0.4910 & 0.6352 & 0.8523 & 0.9545 & 0.9894 \\
170 & 0.0409 & 0.0577 & 0.1119 & 0.2026 & 0.3316 & 0.4824 & 0.6299 & 0.8545 & 0.9563 & 0.9896 \\
180 & 0.0416 & 0.0549 & 0.1039 & 0.1951 & 0.3230 & 0.4744 & 0.6241 & 0.8484 & 0.9556 & 0.9886 \\
190 & 0.0395 & 0.0525 & 0.1015 & 0.1883 & 0.3157 & 0.4650 & 0.6195 & 0.8493 & 0.9542 & 0.9889 \\
200 & 0.0375 & 0.0526 & 0.0983 & 0.1859 & 0.3125 & 0.4629 & 0.6166 & 0.8463 & 0.9526 & 0.9901 \\
\hline
\end{tabular}


Table 6: Powers for BJ with level 0.95 and exact critical values given in Table 1.

\begin{tabular}{|r|r|r|r|r|r|r|r|r|r|r||}
\hline & \multicolumn{10}{|c|}{$\delta \sqrt{k}$} \\
$k$ & 0.1 & 0.5 & 1 & 1.5 & 2 & 2.5 & 3 & 4 & 5 & 6 \\
\hline 20 & 0.0517 & 0.0760 & 0.1371 & 0.2250 & 0.3337 & 0.4364 & 0.5414 & 0.7257 & 0.9268 & 0.9962 \\
30 & 0.0531 & 0.0802 & 0.1379 & 0.2256 & 0.3343 & 0.4512 & 0.5602 & 0.7395 & 0.8561 & 0.9428 \\
40 & 0.0556 & 0.0807 & 0.1414 & 0.2266 & 0.3403 & 0.4575 & 0.5726 & 0.7573 & 0.8745 & 0.9396 \\
50 & 0.0559 & 0.0792 & 0.1421 & 0.2317 & 0.3463 & 0.4663 & 0.5813 & 0.7704 & 0.8885 & 0.9486 \\
60 & 0.0603 & 0.0842 & 0.1433 & 0.2301 & 0.3467 & 0.4710 & 0.5875 & 0.7763 & 0.8950 & 0.9544 \\
70 & 0.0544 & 0.0787 & 0.1357 & 0.2254 & 0.3393 & 0.4669 & 0.5858 & 0.7803 & 0.9028 & 0.9570 \\
80 & 0.0542 & 0.0789 & 0.1362 & 0.2237 & 0.3382 & 0.4664 & 0.5895 & 0.7872 & 0.9070 & 0.9617 \\
90 & 0.0533 & 0.0747 & 0.1332 & 0.2225 & 0.3375 & 0.4647 & 0.5830 & 0.7869 & 0.9068 & 0.9642 \\
100 & 0.0544 & 0.0761 & 0.1351 & 0.2182 & 0.3367 & 0.4598 & 0.5864 & 0.7887 & 0.9098 & 0.9643 \\
110 & 0.0520 & 0.0749 & 0.1310 & 0.2174 & 0.3345 & 0.4605 & 0.5865 & 0.7911 & 0.9130 & 0.9659 \\
120 & 0.0566 & 0.0794 & 0.1362 & 0.2207 & 0.3336 & 0.4648 & 0.5875 & 0.7903 & 0.9156 & 0.9691 \\
130 & 0.0504 & 0.0726 & 0.1297 & 0.2156 & 0.3303 & 0.4583 & 0.5857 & 0.7885 & 0.9147 & 0.9699 \\
140 & 0.0503 & 0.0719 & 0.1290 & 0.2185 & 0.3299 & 0.4627 & 0.5907 & 0.7928 & 0.9192 & 0.9714 \\
150 & 0.0509 & 0.0723 & 0.1277 & 0.2160 & 0.3325 & 0.4615 & 0.5897 & 0.7937 & 0.9197 & 0.9732 \\
160 & 0.0528 & 0.0722 & 0.1263 & 0.2163 & 0.3293 & 0.4580 & 0.5865 & 0.7919 & 0.9169 & 0.9733 \\
170 & 0.0525 & 0.0730 & 0.1295 & 0.2165 & 0.3290 & 0.4566 & 0.5843 & 0.7939 & 0.9209 & 0.9733 \\
180 & 0.0510 & 0.0694 & 0.1228 & 0.2079 & 0.3199 & 0.4468 & 0.5804 & 0.7927 & 0.9206 & 0.9735 \\
190 & 0.0524 & 0.0712 & 0.1228 & 0.2056 & 0.3180 & 0.4498 & 0.5789 & 0.7934 & 0.9180 & 0.9738 \\
200 & 0.0481 & 0.0665 & 0.1186 & 0.2022 & 0.3097 & 0.4429 & 0.5727 & 0.7872 & 0.9182 & 0.9736 \\
\hline
\end{tabular}


Table 7: Powers for BJI with level 0.95 and exact critical values given in Table 1.

\begin{tabular}{||r|r|r|r|r|r|r|r|r|r|r||}
\hline & \multicolumn{10}{|c|}{$\delta \sqrt{k}$} \\
$k$ & 0.1 & 0.5 & 1 & 1.5 & 2 & 2.5 & 3 & 4 & 5 & 6 \\
\hline 20 & 0.0512 & 0.0803 & 0.1534 & 0.2531 & 0.3711 & 0.4896 & 0.5973 & 0.7670 & 0.8664 & 0.9261 \\
30 & 0.0521 & 0.0734 & 0.1418 & 0.2433 & 0.3737 & 0.4989 & 0.6156 & 0.7902 & 0.8962 & 0.9487 \\
40 & 0.0547 & 0.0733 & 0.1325 & 0.2406 & 0.3731 & 0.5065 & 0.6238 & 0.8054 & 0.9094 & 0.9642 \\
50 & 0.0506 & 0.0703 & 0.1310 & 0.2351 & 0.3668 & 0.5016 & 0.6284 & 0.8162 & 0.9201 & 0.9692 \\
60 & 0.0524 & 0.0685 & 0.1254 & 0.2300 & 0.3592 & 0.5077 & 0.6353 & 0.8237 & 0.9286 & 0.9746 \\
70 & 0.0486 & 0.0656 & 0.1196 & 0.2203 & 0.3541 & 0.4996 & 0.6308 & 0.8282 & 0.9348 & 0.9795 \\
80 & 0.0488 & 0.0668 & 0.1179 & 0.2137 & 0.3450 & 0.4978 & 0.6318 & 0.8360 & 0.9372 & 0.9806 \\
90 & 0.0487 & 0.0644 & 0.1185 & 0.2148 & 0.3469 & 0.4954 & 0.6314 & 0.8364 & 0.9422 & 0.9811 \\
100 & 0.0507 & 0.0663 & 0.1154 & 0.2087 & 0.3378 & 0.4907 & 0.6296 & 0.8384 & 0.9410 & 0.9807 \\
110 & 0.0482 & 0.0649 & 0.1166 & 0.2064 & 0.3332 & 0.4843 & 0.6285 & 0.8352 & 0.9458 & 0.9836 \\
120 & 0.0466 & 0.0618 & 0.1109 & 0.2018 & 0.3302 & 0.4744 & 0.6243 & 0.8387 & 0.9449 & 0.9854 \\
130 & 0.0472 & 0.0598 & 0.1048 & 0.1956 & 0.3263 & 0.4756 & 0.6156 & 0.8397 & 0.9485 & 0.9859 \\
140 & 0.0394 & 0.0524 & 0.1000 & 0.1885 & 0.3198 & 0.4664 & 0.6171 & 0.8398 & 0.9480 & 0.9869 \\
150 & 0.0395 & 0.0497 & 0.0963 & 0.1873 & 0.3116 & 0.4614 & 0.6129 & 0.8358 & 0.9479 & 0.9863 \\
160 & 0.0389 & 0.0535 & 0.1010 & 0.1866 & 0.3059 & 0.4549 & 0.6057 & 0.8348 & 0.9463 & 0.9874 \\
170 & 0.0407 & 0.0526 & 0.0969 & 0.1785 & 0.2962 & 0.4460 & 0.5940 & 0.8332 & 0.9466 & 0.9875 \\
180 & 0.0371 & 0.0488 & 0.0884 & 0.1669 & 0.2902 & 0.4377 & 0.5881 & 0.8291 & 0.9468 & 0.9857 \\
190 & 0.0378 & 0.0456 & 0.0879 & 0.1649 & 0.2846 & 0.4284 & 0.5834 & 0.8284 & 0.9449 & 0.9862 \\
200 & 0.0334 & 0.0457 & 0.0843 & 0.1612 & 0.2788 & 0.4238 & 0.5767 & 0.8219 & 0.9428 & 0.9864 \\
\hline
\end{tabular}


Table 8: Powers for SC with level 0.95 and exact critical values given in Table 1.

\begin{tabular}{|r|r|r|r|r|r|r|r|r|r|r||}
\hline & \multicolumn{10}{|c|}{$\delta \sqrt{k}$} \\
$k$ & 0.1 & 0.5 & 1 & 1.5 & 2 & 2.5 & 3 & 4 & 5 & 6 \\
\hline 20 & 0.0493 & 0.0580 & 0.1058 & 0.1888 & 0.3015 & 0.4150 & 0.5160 & 0.6934 & 0.8101 & 0.8859 \\
30 & 0.0470 & 0.0601 & 0.1130 & 0.2089 & 0.3253 & 0.4526 & 0.5673 & 0.7517 & 0.8679 & 0.9280 \\
40 & 0.0501 & 0.0621 & 0.1189 & 0.2149 & 0.3442 & 0.4753 & 0.5947 & 0.7812 & 0.8919 & 0.9525 \\
50 & 0.0490 & 0.0635 & 0.1210 & 0.2253 & 0.3527 & 0.4921 & 0.6097 & 0.8025 & 0.9140 & 0.9645 \\
60 & 0.0512 & 0.0670 & 0.1246 & 0.2238 & 0.3584 & 0.4942 & 0.6259 & 0.8186 & 0.9234 & 0.9722 \\
70 & 0.0526 & 0.0692 & 0.1259 & 0.2317 & 0.3625 & 0.5035 & 0.6398 & 0.8303 & 0.9315 & 0.9773 \\
80 & 0.0533 & 0.0683 & 0.1280 & 0.2332 & 0.3664 & 0.5122 & 0.6488 & 0.8392 & 0.9402 & 0.9811 \\
90 & 0.0521 & 0.0683 & 0.1276 & 0.2342 & 0.3703 & 0.5162 & 0.6537 & 0.8486 & 0.9440 & 0.9828 \\
100 & 0.0537 & 0.0687 & 0.1292 & 0.2370 & 0.3750 & 0.5207 & 0.6561 & 0.8557 & 0.9474 & 0.9844 \\
110 & 0.0550 & 0.0709 & 0.1314 & 0.2362 & 0.3796 & 0.5278 & 0.6608 & 0.8595 & 0.9506 & 0.9864 \\
120 & 0.0521 & 0.0704 & 0.1321 & 0.2377 & 0.3787 & 0.5246 & 0.6673 & 0.8665 & 0.9524 & 0.9871 \\
130 & 0.0528 & 0.0733 & 0.1319 & 0.2336 & 0.3791 & 0.5273 & 0.6693 & 0.8686 & 0.9552 & 0.9882 \\
140 & 0.0543 & 0.0721 & 0.1327 & 0.2314 & 0.3764 & 0.5287 & 0.6736 & 0.8699 & 0.9586 & 0.9885 \\
150 & 0.0529 & 0.0710 & 0.1317 & 0.2319 & 0.3765 & 0.5293 & 0.6745 & 0.8721 & 0.9619 & 0.9900 \\
160 & 0.0533 & 0.0723 & 0.1334 & 0.2325 & 0.3779 & 0.5303 & 0.6760 & 0.8765 & 0.9630 & 0.9908 \\
170 & 0.0552 & 0.0725 & 0.1354 & 0.2330 & 0.3771 & 0.5337 & 0.6742 & 0.8775 & 0.9644 & 0.9920 \\
180 & 0.0535 & 0.0698 & 0.1353 & 0.2341 & 0.3793 & 0.5341 & 0.6771 & 0.8775 & 0.9661 & 0.9931 \\
190 & 0.0555 & 0.0712 & 0.1338 & 0.2357 & 0.3815 & 0.5358 & 0.6777 & 0.8777 & 0.9665 & 0.9938 \\
200 & 0.0540 & 0.0740 & 0.1322 & 0.2380 & 0.3792 & 0.5431 & 0.6792 & 0.8826 & 0.9688 & 0.9936 \\
\hline
\end{tabular}


Table 9: Powers for SCI with level 0.95 and asymptotic critical value 0.456.

\begin{tabular}{||r|r|r|r|r|r|r|r|r|r|r||}
\hline & \multicolumn{10}{|c|}{$\delta \sqrt{k}$} \\
$k$ & 0.1 & 0.5 & 1 & 1.5 & 2 & 2.5 & 3 & 4 & 5 & 6 \\
\hline 20 & 0.0456 & 0.0802 & 0.1568 & 0.2693 & 0.3972 & 0.5077 & 0.6110 & 0.7711 & 0.8651 & 0.9226 \\
30 & 0.0503 & 0.0803 & 0.1631 & 0.2812 & 0.4161 & 0.5464 & 0.6530 & 0.8170 & 0.9088 & 0.9546 \\
40 & 0.0522 & 0.0804 & 0.1623 & 0.2883 & 0.4310 & 0.5640 & 0.6802 & 0.8429 & 0.9276 & 0.9708 \\
50 & 0.0511 & 0.0825 & 0.1665 & 0.2900 & 0.4368 & 0.5774 & 0.6924 & 0.8583 & 0.9442 & 0.9793 \\
60 & 0.0530 & 0.0818 & 0.1637 & 0.2933 & 0.4385 & 0.5846 & 0.7038 & 0.8709 & 0.9510 & 0.9846 \\
70 & 0.0549 & 0.0822 & 0.1702 & 0.2953 & 0.4425 & 0.5922 & 0.7150 & 0.8814 & 0.9587 & 0.9860 \\
80 & 0.0541 & 0.0838 & 0.1690 & 0.2943 & 0.4489 & 0.5964 & 0.7258 & 0.8880 & 0.9625 & 0.9887 \\
90 & 0.0511 & 0.0841 & 0.1647 & 0.2941 & 0.4475 & 0.5998 & 0.7297 & 0.8963 & 0.9662 & 0.9900 \\
100 & 0.0549 & 0.0837 & 0.1660 & 0.2935 & 0.4514 & 0.5983 & 0.7348 & 0.8989 & 0.9690 & 0.9906 \\
110 & 0.0574 & 0.0809 & 0.1624 & 0.2978 & 0.4517 & 0.6050 & 0.7372 & 0.9024 & 0.9697 & 0.9922 \\
120 & 0.0553 & 0.0837 & 0.1664 & 0.2969 & 0.4484 & 0.6088 & 0.7389 & 0.9070 & 0.9711 & 0.9930 \\
130 & 0.0558 & 0.0874 & 0.1658 & 0.2869 & 0.4517 & 0.6113 & 0.7409 & 0.9083 & 0.9730 & 0.9930 \\
140 & 0.0535 & 0.0850 & 0.1635 & 0.2873 & 0.4512 & 0.6069 & 0.7427 & 0.9110 & 0.9773 & 0.9942 \\
150 & 0.0545 & 0.0848 & 0.1655 & 0.2877 & 0.4467 & 0.6070 & 0.7460 & 0.9136 & 0.9772 & 0.9945 \\
160 & 0.0549 & 0.0806 & 0.1643 & 0.2890 & 0.4470 & 0.6109 & 0.7429 & 0.9144 & 0.9786 & 0.9946 \\
170 & 0.0554 & 0.0814 & 0.1639 & 0.2895 & 0.4484 & 0.6070 & 0.7464 & 0.9168 & 0.9803 & 0.9957 \\
180 & 0.0546 & 0.0833 & 0.1629 & 0.2918 & 0.4502 & 0.6083 & 0.7451 & 0.9168 & 0.9802 & 0.9961 \\
190 & 0.0533 & 0.0827 & 0.1647 & 0.2915 & 0.4522 & 0.6148 & 0.7428 & 0.9196 & 0.9814 & 0.9967 \\
200 & 0.0544 & 0.0813 & 0.1620 & 0.2937 & 0.4530 & 0.6118 & 0.7442 & 0.9216 & 0.9822 & 0.9960 \\
\hline
\end{tabular}


Table 10: Powers for KS with level 0.95 and asymptotic critical value 1.067.

\begin{tabular}{||r|r|r|r|r|r|r|r|r|r|r||}
\hline & \multicolumn{10}{|c|}{$\delta \sqrt{k}$} \\
$k$ & 0.1 & 0.5 & 1 & 1.5 & 2 & 2.5 & 3 & 4 & 5 & 6 \\
\hline 20 & 0.0425 & 0.0560 & 0.1046 & 0.1903 & 0.2965 & 0.4049 & 0.5132 & 0.6883 & 0.8136 & 0.9313 \\
30 & 0.0414 & 0.0581 & 0.1089 & 0.1976 & 0.3016 & 0.4278 & 0.5437 & 0.7326 & 0.8591 & 0.9230 \\
40 & 0.0467 & 0.0628 & 0.1128 & 0.1995 & 0.3084 & 0.4402 & 0.5590 & 0.7511 & 0.8791 & 0.9462 \\
50 & 0.0476 & 0.0631 & 0.1146 & 0.1992 & 0.3143 & 0.4416 & 0.5683 & 0.7681 & 0.8949 & 0.9568 \\
60 & 0.0481 & 0.0643 & 0.1151 & 0.1968 & 0.3167 & 0.4487 & 0.5746 & 0.7812 & 0.9023 & 0.9639 \\
70 & 0.0504 & 0.0652 & 0.1152 & 0.2016 & 0.3220 & 0.4514 & 0.5824 & 0.7901 & 0.9139 & 0.9688 \\
80 & 0.0512 & 0.0683 & 0.1148 & 0.1977 & 0.3226 & 0.4558 & 0.5896 & 0.7987 & 0.9203 & 0.9727 \\
90 & 0.0508 & 0.0671 & 0.1138 & 0.2001 & 0.3194 & 0.4557 & 0.5940 & 0.8095 & 0.9229 & 0.9754 \\
100 & 0.0524 & 0.0691 & 0.1149 & 0.2056 & 0.3209 & 0.4534 & 0.5945 & 0.8127 & 0.9268 & 0.9768 \\
110 & 0.0526 & 0.0684 & 0.1134 & 0.2010 & 0.3215 & 0.4523 & 0.5947 & 0.8168 & 0.9285 & 0.9772 \\
120 & 0.0534 & 0.0665 & 0.1160 & 0.1996 & 0.3191 & 0.4570 & 0.5949 & 0.8205 & 0.9330 & 0.9793 \\
130 & 0.0508 & 0.0682 & 0.1179 & 0.2006 & 0.3156 & 0.4535 & 0.5958 & 0.8189 & 0.9342 & 0.9798 \\
140 & 0.0504 & 0.0672 & 0.1145 & 0.1949 & 0.3121 & 0.4529 & 0.5938 & 0.8161 & 0.9345 & 0.9817 \\
150 & 0.0505 & 0.0658 & 0.1123 & 0.1969 & 0.3073 & 0.4495 & 0.5911 & 0.8148 & 0.9381 & 0.9836 \\
160 & 0.0526 & 0.0676 & 0.1130 & 0.1954 & 0.3115 & 0.4485 & 0.5928 & 0.8196 & 0.9387 & 0.9844 \\
170 & 0.0530 & 0.0692 & 0.1129 & 0.1961 & 0.3100 & 0.4472 & 0.5934 & 0.8191 & 0.9418 & 0.9848 \\
180 & 0.0526 & 0.0663 & 0.1139 & 0.1963 & 0.3127 & 0.4489 & 0.5918 & 0.8183 & 0.9427 & 0.9857 \\
190 & 0.0524 & 0.0673 & 0.1139 & 0.1967 & 0.3142 & 0.4508 & 0.5939 & 0.8172 & 0.9410 & 0.9858 \\
200 & 0.0531 & 0.0680 & 0.1127 & 0.1974 & 0.3131 & 0.4513 & 0.5924 & 0.8155 & 0.9430 & 0.9856 \\
\hline
\end{tabular}


Table 11: Powers for KSI with level 0.95 and asymptotic critical value 0.220.

\begin{tabular}{||r|r|r|r|r|r|r|r|r|r|r||}
\hline & \multicolumn{10}{|c|}{$\delta \sqrt{k}$} \\
$k$ & 0.1 & 0.5 & 1 & 1.5 & 2 & 2.5 & 3 & 4 & 5 & 6 \\
\hline 20 & 0.0431 & 0.0655 & 0.1326 & 0.2302 & 0.3546 & 0.4717 & 0.5736 & 0.7432 & 0.8473 & 0.9154 \\
30 & 0.0471 & 0.0667 & 0.1345 & 0.2372 & 0.3659 & 0.4935 & 0.6098 & 0.7876 & 0.8908 & 0.9464 \\
40 & 0.0514 & 0.0690 & 0.1316 & 0.2389 & 0.3708 & 0.5074 & 0.6305 & 0.8068 & 0.9120 & 0.9628 \\
50 & 0.0497 & 0.0710 & 0.1346 & 0.2415 & 0.3734 & 0.5140 & 0.6396 & 0.8236 & 0.9265 & 0.9738 \\
60 & 0.0521 & 0.0733 & 0.1310 & 0.2407 & 0.3779 & 0.5204 & 0.6482 & 0.8359 & 0.9330 & 0.9784 \\
70 & 0.0516 & 0.0735 & 0.1362 & 0.2429 & 0.3788 & 0.5211 & 0.6544 & 0.8473 & 0.9432 & 0.9804 \\
80 & 0.0517 & 0.0733 & 0.1350 & 0.2386 & 0.3789 & 0.5296 & 0.6621 & 0.8547 & 0.9477 & 0.9849 \\
90 & 0.0490 & 0.0698 & 0.1341 & 0.2422 & 0.3784 & 0.5297 & 0.6675 & 0.8616 & 0.9497 & 0.9861 \\
100 & 0.0516 & 0.0725 & 0.1353 & 0.2414 & 0.3798 & 0.5285 & 0.6649 & 0.8636 & 0.9539 & 0.9864 \\
110 & 0.0546 & 0.0723 & 0.1315 & 0.2408 & 0.3774 & 0.5282 & 0.6674 & 0.8652 & 0.9564 & 0.9872 \\
120 & 0.0495 & 0.0721 & 0.1340 & 0.2357 & 0.3764 & 0.5285 & 0.6718 & 0.8711 & 0.9579 & 0.9875 \\
130 & 0.0520 & 0.0758 & 0.1345 & 0.2317 & 0.3742 & 0.5331 & 0.6716 & 0.8719 & 0.9603 & 0.9891 \\
140 & 0.0500 & 0.0745 & 0.1321 & 0.2312 & 0.3684 & 0.5288 & 0.6722 & 0.8722 & 0.9629 & 0.9902 \\
150 & 0.0498 & 0.0725 & 0.1319 & 0.2304 & 0.3690 & 0.5254 & 0.6744 & 0.8756 & 0.9656 & 0.9914 \\
160 & 0.0502 & 0.0711 & 0.1305 & 0.2327 & 0.3685 & 0.5273 & 0.6752 & 0.8797 & 0.9646 & 0.9918 \\
170 & 0.0524 & 0.0692 & 0.1329 & 0.2320 & 0.3721 & 0.5265 & 0.6712 & 0.8756 & 0.9657 & 0.9930 \\
180 & 0.0526 & 0.0706 & 0.1313 & 0.2319 & 0.3734 & 0.5270 & 0.6718 & 0.8772 & 0.9667 & 0.9942 \\
190 & 0.0514 & 0.0725 & 0.1310 & 0.2361 & 0.3696 & 0.5298 & 0.6731 & 0.8776 & 0.9678 & 0.9944 \\
200 & 0.0505 & 0.0732 & 0.1295 & 0.2366 & 0.3697 & 0.5275 & 0.6705 & 0.8772 & 0.9698 & 0.9933 \\
\hline
\end{tabular}


Table 12: Powers for BJI with level 0.95 and asymptotic critical value 1.313.

\begin{tabular}{|r|r|r|r|r|r|r|r|r|r|r||}
\hline & \multicolumn{10}{|c|}{$\delta \sqrt{k}$} \\
$k$ & 0.1 & 0.5 & 1 & 1.5 & 2 & 2.5 & 3 & 4 & 5 & 6 \\
\hline 20 & 0.0285 & 0.0471 & 0.1079 & 0.2006 & 0.3180 & 0.4374 & 0.5436 & 0.7191 & 0.8370 & 0.9084 \\
30 & 0.0380 & 0.0563 & 0.1141 & 0.2070 & 0.3325 & 0.4597 & 0.5829 & 0.7668 & 0.8791 & 0.9398 \\
40 & 0.0425 & 0.0550 & 0.1109 & 0.2098 & 0.3382 & 0.4743 & 0.6000 & 0.7875 & 0.9015 & 0.9579 \\
50 & 0.0433 & 0.0612 & 0.1114 & 0.2137 & 0.3388 & 0.4791 & 0.6106 & 0.8044 & 0.9171 & 0.9688 \\
60 & 0.0463 & 0.0611 & 0.1139 & 0.2091 & 0.3451 & 0.4863 & 0.6190 & 0.8141 & 0.9249 & 0.9730 \\
70 & 0.0484 & 0.0642 & 0.1196 & 0.2183 & 0.3462 & 0.4892 & 0.6289 & 0.8285 & 0.9365 & 0.9768 \\
80 & 0.0463 & 0.0620 & 0.1180 & 0.2142 & 0.3456 & 0.4948 & 0.6345 & 0.8400 & 0.9406 & 0.9816 \\
90 & 0.0466 & 0.0604 & 0.1177 & 0.2122 & 0.3445 & 0.4945 & 0.6359 & 0.8438 & 0.9434 & 0.9830 \\
100 & 0.0497 & 0.0667 & 0.1159 & 0.2132 & 0.3453 & 0.4953 & 0.6325 & 0.8487 & 0.9478 & 0.9832 \\
110 & 0.0506 & 0.0664 & 0.1145 & 0.2107 & 0.3479 & 0.4971 & 0.6400 & 0.8453 & 0.9493 & 0.9850 \\
120 & 0.0477 & 0.0641 & 0.1196 & 0.2124 & 0.3435 & 0.4910 & 0.6421 & 0.8556 & 0.9507 & 0.9860 \\
130 & 0.0506 & 0.0672 & 0.1193 & 0.2069 & 0.3403 & 0.4984 & 0.6400 & 0.8550 & 0.9533 & 0.9868 \\
140 & 0.0488 & 0.0650 & 0.1166 & 0.2072 & 0.3361 & 0.4948 & 0.6398 & 0.8541 & 0.9575 & 0.9889 \\
150 & 0.0490 & 0.0632 & 0.1173 & 0.2057 & 0.3354 & 0.4914 & 0.6429 & 0.8569 & 0.9582 & 0.9900 \\
160 & 0.0498 & 0.0667 & 0.1170 & 0.2103 & 0.3415 & 0.4901 & 0.6443 & 0.8603 & 0.9564 & 0.9901 \\
170 & 0.0508 & 0.0652 & 0.1188 & 0.2129 & 0.3404 & 0.4944 & 0.6423 & 0.8611 & 0.9582 & 0.9914 \\
180 & 0.0509 & 0.0656 & 0.1175 & 0.2120 & 0.3430 & 0.4968 & 0.6405 & 0.8600 & 0.9623 & 0.9929 \\
190 & 0.0510 & 0.0666 & 0.1178 & 0.2118 & 0.3437 & 0.4966 & 0.6459 & 0.8603 & 0.9629 & 0.9927 \\
200 & 0.0490 & 0.0690 & 0.1183 & 0.2148 & 0.3396 & 0.4964 & 0.6441 & 0.8611 & 0.9638 & 0.9910 \\
\hline
\end{tabular}




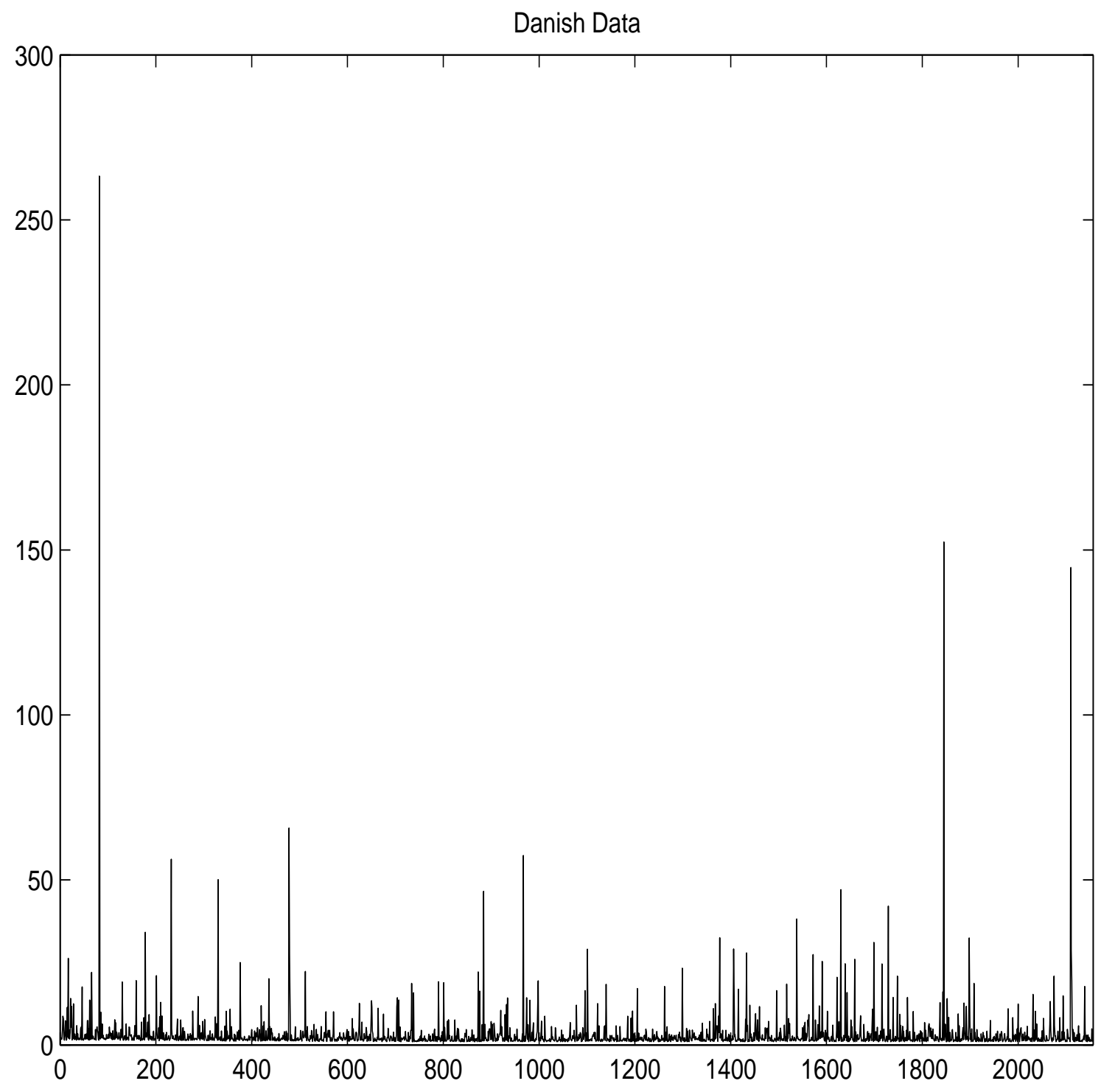

Figure 1: Danish fire loss data. This consists of 2156 losses over one million Danish Krone (DKK) from the years 1980 to 1990, inclusive. 
sc
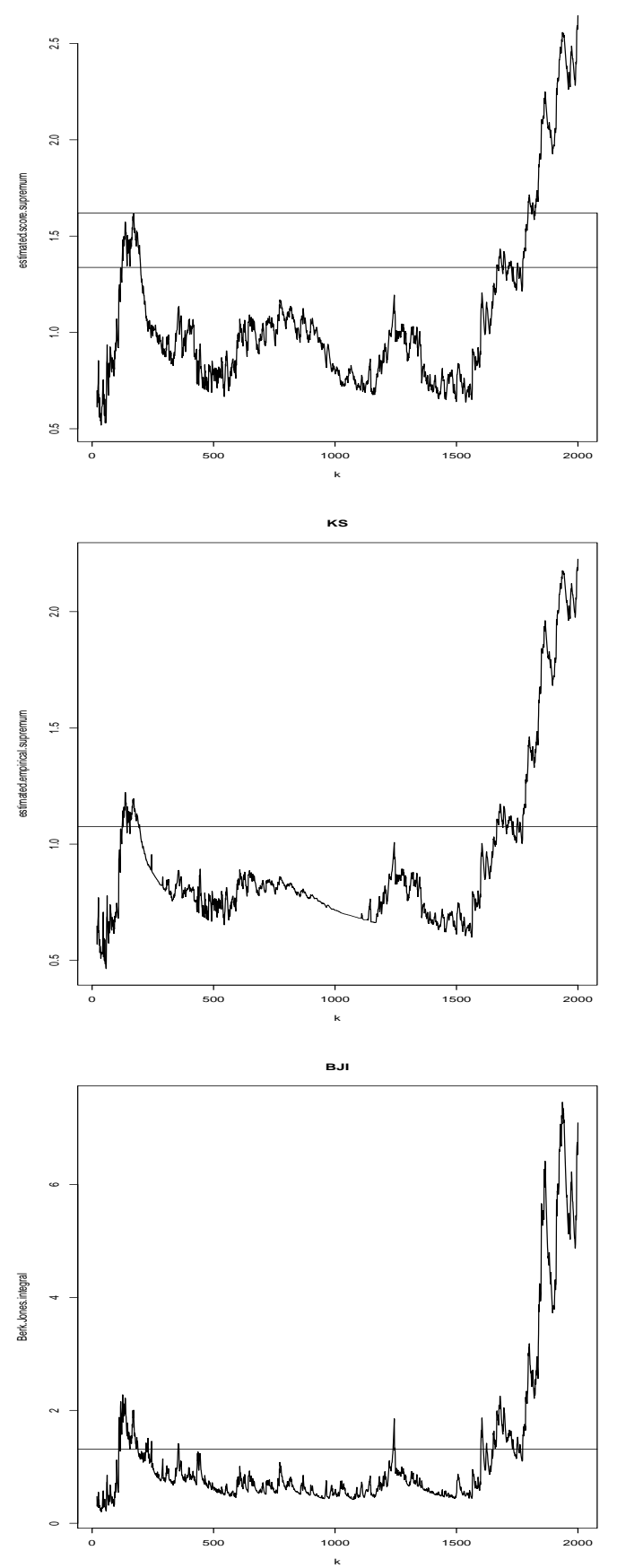

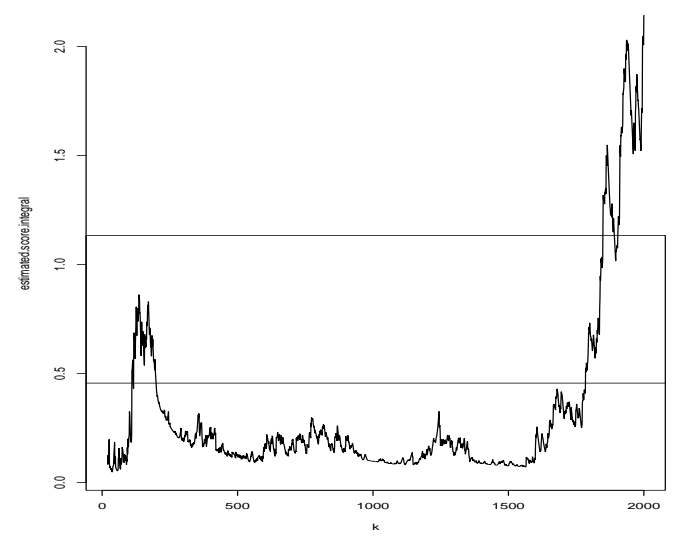

ksı
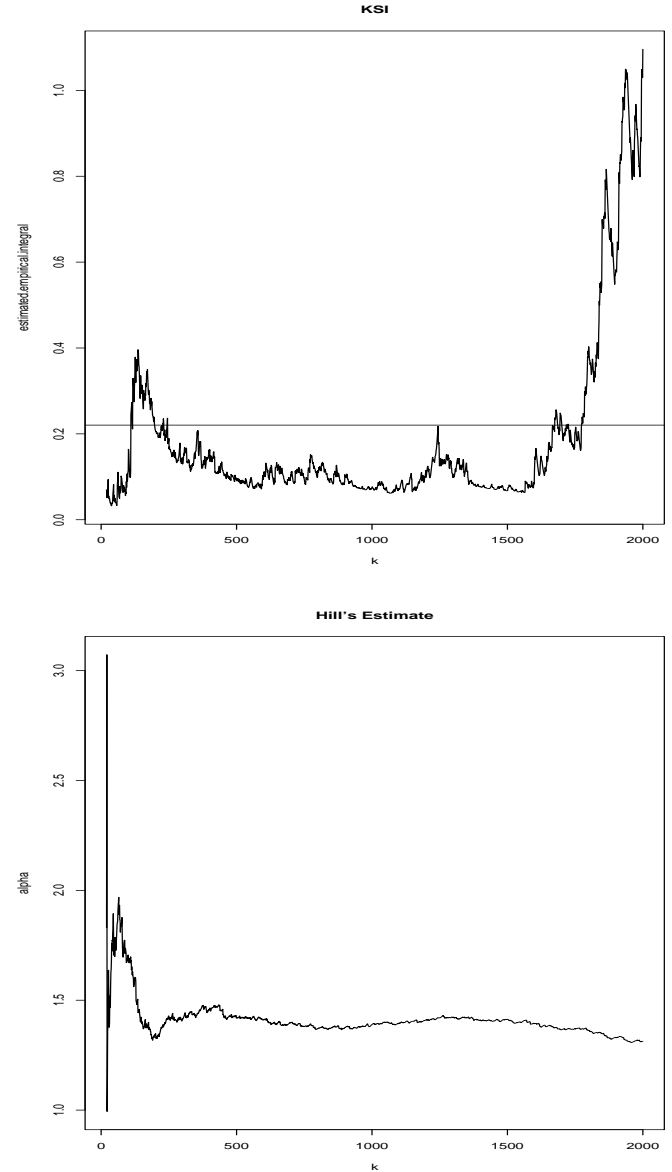

Figure 2: Analysis of the Danish fire loss data. 

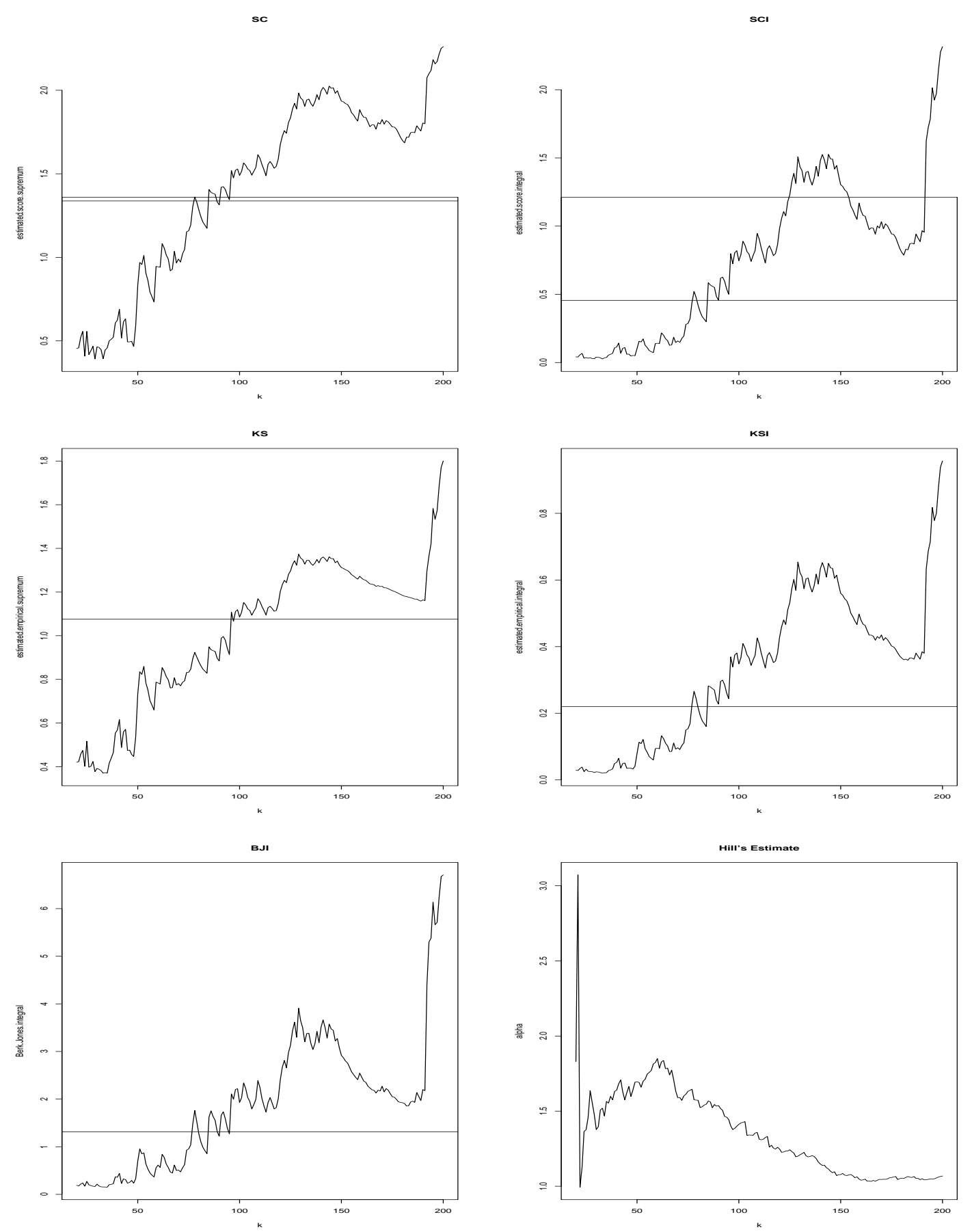

Figure 3: Analysis of the block maxima of the Danish fire loss data. 


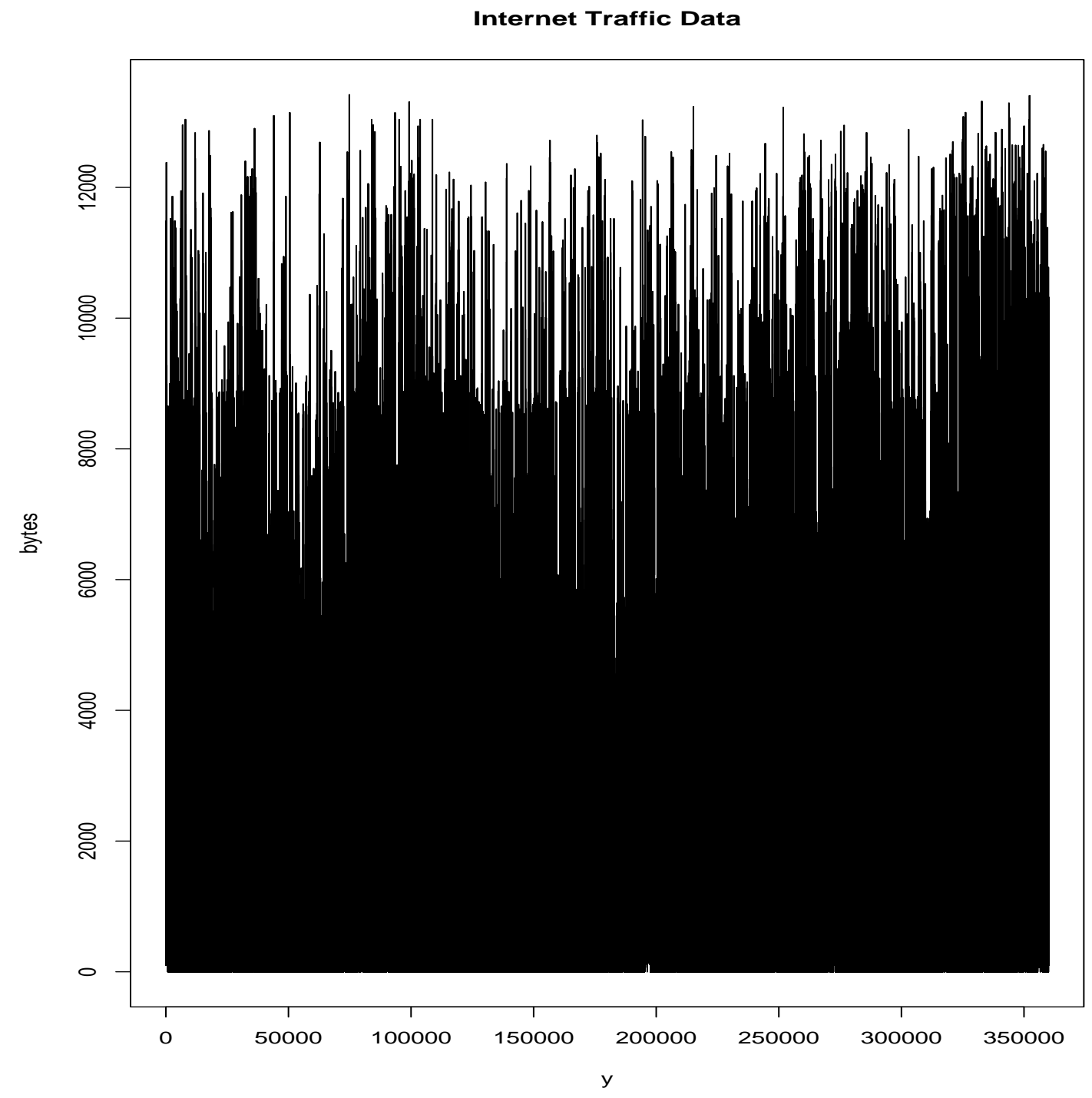

Figure 4: Internet traffic data. The Ethernet series used here are part of a data set collected at Bellcore in August of 1989. They correspond to one "normal" hour's worth of traffic, collected every 10 milliseconds, thus resulting in a length of 360,000 . This data set measures the number of bytes per unit time. 

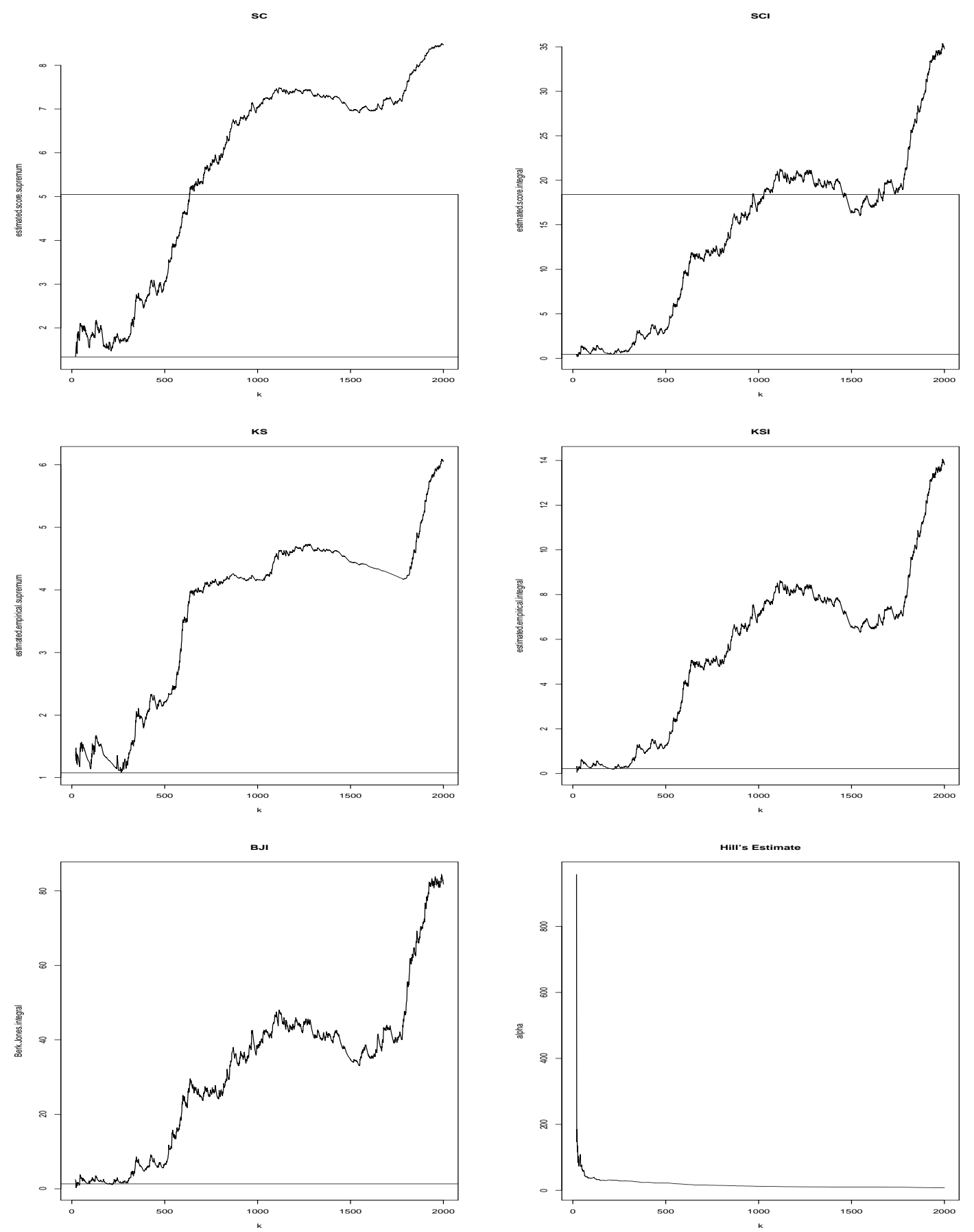

Figure 5: Analysis of the internet traffic data data. 
sc
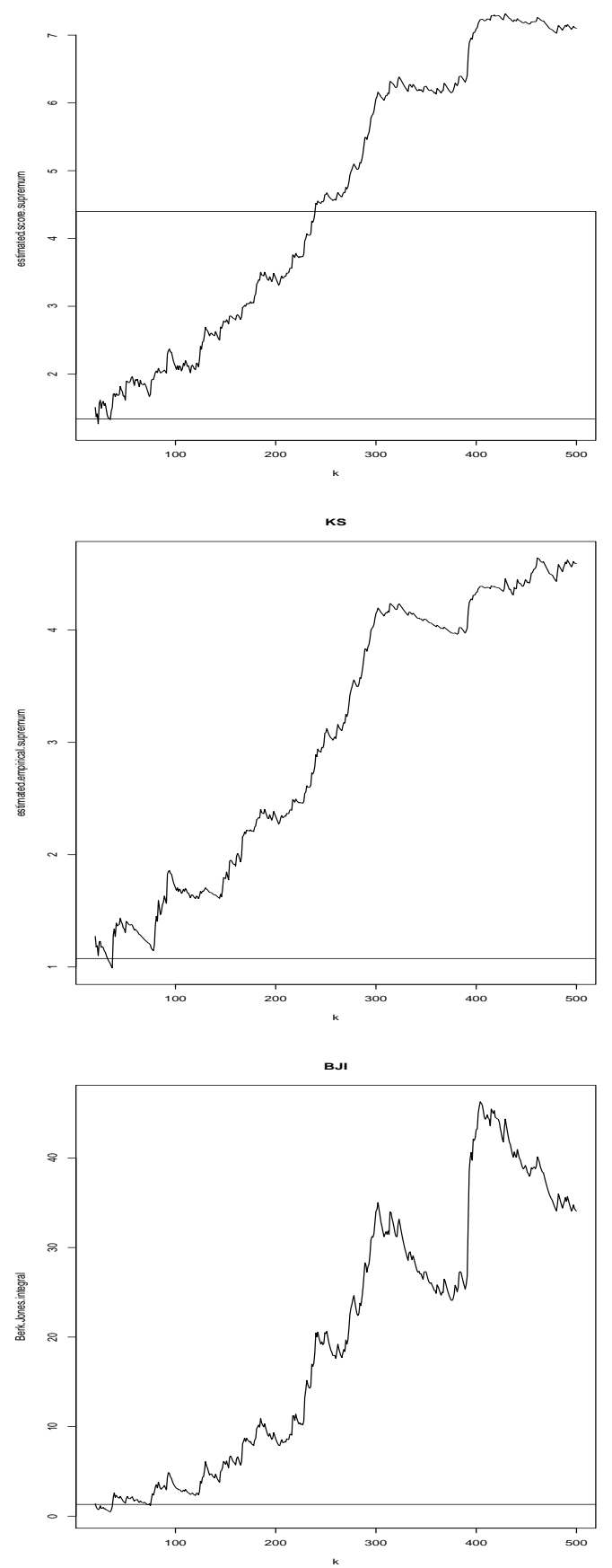

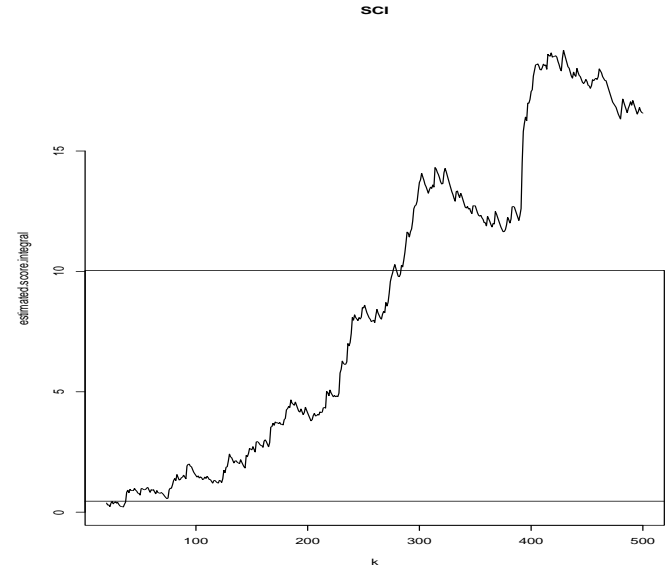

ksı

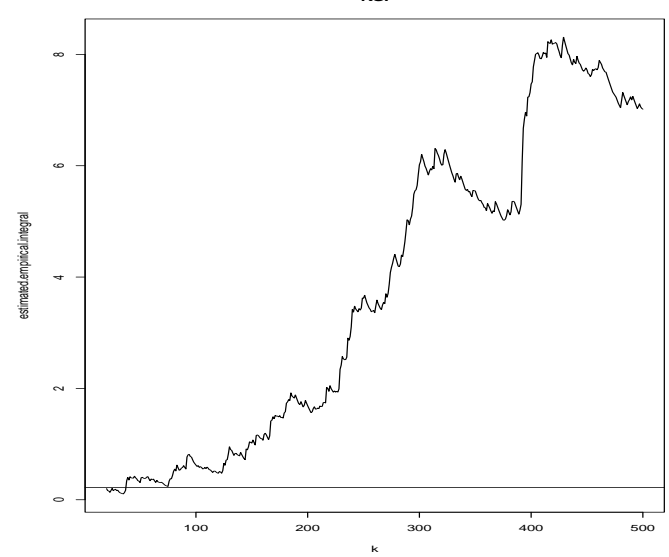

Hill's Estimate

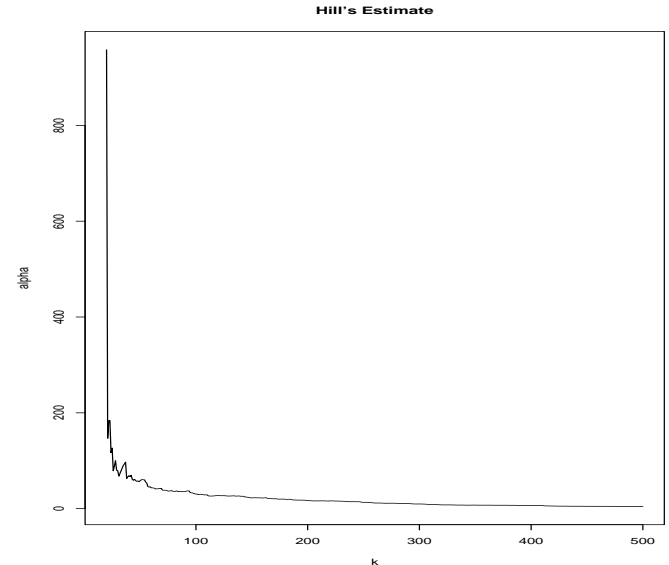

Figure 6: Analysis of the block maxima of the internet traffic data. 